\title{
Anti-spike IgG causes severe acute lung injury by skewing macrophage responses during acute SARS-CoV infection
}

\author{
Li Liu, ${ }^{1,2}$ Qiang Wei, ${ }^{3}$ Qingqing Lin, Jun Fang, ${ }^{1}$ Haibo Wang, ${ }^{1}$ Hauyee Kwok, ${ }^{1}$ Hangying Tang, \\ Kenji Nishiura, ${ }^{1}$ Jie Peng, ${ }^{1}$ Zhiwu Tan, ${ }^{1}$ Tongjin Wu, ${ }^{1}$ Ka-Wai Cheung, ${ }^{1}$ Kwok-Hung Chan, ${ }^{1}$ \\ Xavier Alvarez, ${ }^{4}$ Chuan Qin, ${ }^{3}$ Andrew Lackner, ${ }^{4}$ Stanley Perlman, ${ }^{5,6}$ Kwok-Yung Yuen, ${ }^{1}$ \\ and Zhiwei Chen ${ }^{1,2}$ \\ 'AIDS Institute and Department of Microbiology, State Key Laboratory of Emerging Infectious Disease, Li Ka Shing \\ Faculty of Medicine, The University of Hong Kong, Hong Kong, China. ${ }^{2} \mathrm{HKU}-$ AIDS Institute Shenzhen Research Laboratory \\ and AIDS Clinical Research Laboratory, Shenzhen Key Laboratory of Infection and Immunity, Shenzhen Key Clinical \\ Department of Emerging Infectious Diseases, Shenzhen Third People's Hospital, Shenzhen, China. ${ }^{3}$ Institute of Laboratory \\ Animal Science, Chinese Academy of Medical Sciences (CAMS) and Peking Union Medical College (PUMC), Beijing, China. \\ ${ }^{4}$ Division of Comparative Pathology, Tulane National Primate Research Center, Covington, Louisiana, USA. ${ }^{5}$ Department \\ of Microbiology and Immunology, University of lowa, lowa City, lowa, USA. ${ }^{6}$ State Key Laboratory of Respiratory Disease, \\ National Clinical Research Center for Respiratory Disease, Guangzhou Institute of Respiratory Health, the First Affiliated \\ Hospital of Guangzhou Medical University, Guangzhou, China.
}

\begin{abstract}
Authorship note: AL is deceased.
Conflict of interest: This work was financially supported by grants from the US NIH R01HL080211 and HK RGC TRS T11-706/18-N to ZC, the TNPRC (base grant RR00164), R01060699 to SP, HMRF 16150662, and the University Development Fund/Li Ka Shing Faculty of Medicine Matching Fund of the University of Hong Kong to its AIDS Institute and Hong Kong RGV.
\end{abstract}

License: Copyright 2019, American Society for Clinical Investigation.

Submitted: July 23, 2018

Accepted: January 11, 2019

Published: February 21, 2019

Reference information: JCI Insight. 2019;4(4):e123158. https://doi.org/10.1172/jici. insight.123158.
Newly emerging viruses, such as severe acute respiratory syndrome coronavirus (SARS-CoV), Middle Eastern respiratory syndrome CoVs (MERS-CoV), and H7N9, cause fatal acute lung injury (ALI) by driving hypercytokinemia and aggressive inflammation through mechanisms that remain elusive. In SARS-CoV/macaque models, we determined that anti-spike IgC (S-IgC), in productively infected lungs, causes severe ALI by skewing inflammation-resolving response. Alveolar macrophages underwent functional polarization in acutely infected macaques, demonstrating simultaneously both proinflammatory and wound-healing characteristics. The presence of S-IgC prior to viral clearance, however, abrogated wound-healing responses and promoted MCP1 and IL-8 production and proinflammatory monocyte/macrophage recruitment and accumulation. Critically, patients who eventually died of SARS (hereafter referred to as deceased patients) displayed similarly accumulated pulmonary proinflammatory, absence of wound-healing macrophages, and faster neutralizing antibody responses. Their sera enhanced SARS-CoV-induced MCP1 and IL-8 production by human monocyte-derived wound-healing macrophages, whereas blockade of $\mathrm{Fc} \gamma \mathrm{R}$ reduced such effects. Our findings reveal a mechanism responsible for virus-mediated ALI, define a pathological consequence of viral specific antibody response, and provide a potential target for treatment of SARS-CoV or other virus-mediated lung injury.

\section{Introduction}

Severe acute respiratory syndrome coronavirus (SARS-CoV) causes fatal human respiratory disease (1-3). Patients with SARS (hereafter referred to as SARS patients) displayed the characteristics of acute lung injury (ALI), including diffuse alveolar damage (DAD), epithelial necrosis, and fibrin and hyaline deposition $(4,5)$. Most patients who die of SARS develop acute respiratory distress syndrome (ARDS), the most severe form of ALI $(6,7)$. Recent outbreaks of severe acute respiratory infections of emerging viruses, including Middle Eastern respiratory syndrome CoVs (MERS-CoV), highly pathogenic avian influenza viruses (e.g., H5N1 and H7N9), highlight the need to characterize the mechanisms responsible for virus-mediated ALI or ARDS.

Fundamental to ARDS is the acute onset of lung inflammation, which is intimately tied to monocyte/ macrophage polarization and function $(7,8)$. Lung macrophages are highly plastic and heterogeneous cells that are resident in the lung interstitium and alveoli or recruited upon inflammatory stimuli. Inflammatory monocytes and resident tissue macrophages play critical roles in initiating and maintaining inflammation 
during the acute stage of ARDS, as well as in the resolution phages of inflammation and recovery from ARDS. At steady state, resident macrophages are normally quiescent to prevent damaging the alveoli and are critically involved in normal tissue homeostasis. After tissue injury or during infection, resident macrophages become activated. Circulating monocytes can be efficiently recruited to the site of injury. Inflammatory monocytes/macrophages (IMMs) and resident macrophages undergo marked phenotypic and functional changes, and they can be classified into proinflammatory (M1 or classically activated) and inflammatory-resolving (M2, alternatively activated, wound-healing, or antiinflammatory) macrophages, with a continuum of macrophage polarization existing beyond these discrete categories (9). During acute infection, monocytes/macrophages often display a phenotype of classically activated macrophages. These cells mediate host defenses against viruses and also promote lung injury by producing nitric oxide (NO); ROS; IL-1, IL-6, and IL-8; and TNF. Simultaneously, some macrophages may become alternatively activated, exerting antiinflammatory function and regulating wound healing by producing matrix metalloproteinases (MMPs), growth factors, and antiinflammatory cytokines, particularly TGF- $\beta$. When the pathogen or inflammatory stimulus is eliminated, proinflammatory macrophages diminish. The predominant macrophage population assumes a wound-healing phenotype. At the final recovery stage, macrophages show a regulatory/suppressive phenotype, secreting increased levels of IL-10, which facilitates the resolution of wound healing and restores homeostasis. When the wound-healing response is well organized and controlled, the inflammatory response resolves quickly, and normal tissue architecture is restored. Disturbances in wound-healing response can lead to uncontrolled production of inflammatory mediators, contributing to a state of persistent injury (9-11). In patients who eventually died of SARS (hereafter referred to as deceased patients) and animal models, extensive lung damage is associated with high initial viral loads, increased IMM accumulation in the lungs, and elevated serum proinflammatory cytokine (IL-1, IL-6, IL-8, CXCL-10, and MCP1) levels (4, $5,12,13)$. While much is known about the terminal phase of SARS, little is known about the early immune events during the acute phase of infection. Studies defining macrophage heterogeneity and function during acute infection and ALI using nonhuman primate and patient specimens are limited, and the factors driving the hypercytokinemia and aggressive inflammation remain elusive.

During the SARS outbreak in Hong Kong, most patients (70\%-80\%) presented with abnormal chest radiographs, with approximately one-quarter of these individuals progressing to ALI. After 12 days, 80\% of these SARS patients developed ARDS, coincident with IgG seroconversion (6). In a detailed analysis of antibody responses against SARS-CoV spike (S) glycoprotein, we reported that the anti-S neutralizing antibody $(\mathrm{NAb})$ response developed significantly faster in deceased patients compared with recovered patients after the onset of clinical symptoms (14). It took an average of 20 days for the recovered patients to reach their peak of NAb activities, as opposed to only 14.7 days for the deceased patients. Moreover, the actual $\mathrm{NAb}$ titer is significantly higher in deceased patients compared with that in the recovered patients during the same time period (14). These findings suggest a role of anti-S antibodies in SARS-CoV-mediated ALI during acute infection. Consistently, preexisting serum antibodies against influenza antigens were found to associate with worse clinical severity and poor outcomes in patients during the 2009 influenza pandemic $(15,16)$. Moreover, multiple vaccine platforms and viral infection appeared to induce SARS-CoV-specific immune memory that enhanced lung inflammation following homologous challenge in mice and African green monkeys (17-19). The mechanism responsible for the immunopathologic reaction remains elusive. Recent studies suggested that $\mathrm{T}$ cells play a crucial role in protection of mice against lethal SARS-CoV infection (20-22). Enhanced pulmonary immunopathology in vaccinated and challenged animals reflects an inadequate Th1 response $(22,23)$. The role of virus-specific antibody response in SARS-CoV-induced lung injury has yet to be clearly defined. Therefore, we used vaccination and anti-S-IgG passive immunization strategies to evaluate the effects of anti-S antibodies on SARS-CoV-induced ALI in Chinese rhesus monkeys (Macaca mulatta). Our results now show that, in productively infected lungs, anti-S-IgG causes severe ALI by skewing inflammatory resolving responses during acute infection.

\section{Results}

Vaccine-induced S-specific immunity resulted in severe ALI in SARS-CoV infected Chinese macaques. We initially compared the pathological changes in the lungs of rhesus macaques vaccinated with a modified vaccinia Ankara (MVA) virus encoding full-length SARS-CoV S glycoprotein (ADS-MVA) or control at 7 and 35 days after pathogenic SARS-CoV ${ }_{\text {PUMC }}\left(1 \times 10^{5}\right.$ tissue culture infectious dose of $50 \%$ [TCID $\left.\left._{50}\right]\right)$ challenge (24). Three healthy macaques were included as controls. Sixteen macaques were i.m. immunized twice: 8 animals with 
ADS-MVA and another 8 animals with a control MVA (ADC-MVA) (Figure 1A) (24). Vaccination with ADS-MVA induced high levels of anti-SARS-CoV NAbs in all 8 macaques, with sera $\mathrm{IC}_{50}$ values ranging from 10,232- to 28,703 -fold dilutions (Figure 1B). None of the macaques developed fever during or after vaccination (normal body temperature $37.8^{\circ} \mathrm{C}-38.1^{\circ} \mathrm{C}$ ). The $\mathrm{IC}_{50}$ values of these sera were maintained, with an over-2,000-fold reciprocal serum dilution at 4 weeks after the secondary immunization, a time when all of the animals were i.n. challenged (Figure 1B). After viral challenge, all macaques developed fever between 1 and 5 days after inoculation (dpi) ranging from $38.6^{\circ} \mathrm{C}-39.2^{\circ} \mathrm{C}$, and sera from control vaccinated macaques showed increased neutralizing activity after $14 \mathrm{dpi}$, indicating establishment of infection (Figure 1B). SARS CoV RNA was readily detected on oral swabs from all control macaques examined at multiple time points using real-time reverse transcription PCR (real-time RT-PCR) but only in 3 of the 8 ADS-MVA-vaccinated macaques with lower NAb titers at 2 dpi (SL11, SE13, and SL14) (Figure 1C), suggesting reduced productive viral infection in the immunized macaques through S-specific immunity.

However, histological examination revealed acute DAD with various degrees of severity in 6 ADSMVA-vaccinated macaques at 7 and 35 dpi, whereas most control macaques in the ADC-MVA group showed minor to moderate inflammation (Figure 1D). To better characterize the pathological changes, we adopted a 6-grade scoring system to describe the severity of the lung damage from least severe to most severe: $0,-; 1,+; 2,++; 3,+++; 4,++++;$ and $5,+++++$ (Supplemental Figure 1; supplemental material available online with this article; https://doi.org/10.1172/jci.insight.123158DS1). In the control vaccinated group, 5 macaques showed minor inflammation with slight septa broadening and inflammatory cells infiltration that we scored as + (CL23, CE25, CE20, CE21, and CL19; Figure 1D, Supplemental Figure 1B, and Supplemental Figure 2). Two macaques showed moderate inflammation with more interstitial mononuclear inflammatory infiltration and were scored as ++ (CL26, CE24; Supplemental Figure 1C and Supplemental Figure 2). Only 1 macaque showed typical symptoms of acute DAD with extensive exudation and cell infiltration at 35 dpi (CL22, scored as ++++; Supplemental Figure 2). In the ADS-MVA-vaccinated group, 4 macaques showed typical symptom of acute DAD with extensive exudation and many-cell infiltration in alveolar cavities (SL11, SE12, SL16, and SL18, scored as ++++ ; Supplemental Figure 1E and Supplemental Figure 2). One macaque showed severe acute DAD with exudation, hyaline membrane formation along the alveoli, pneumocyte desquamation, and damaged alveolus filled with hemorrhage and inflammatory cells (SL15, scored as +++++ ; Figure 1D). One macaque showed early symptom of acute DAD (SL14, scored as +++; Supplemental Figure 1D), and the remaining 2 macaques showed moderate inflammation (SE13 and SE17, scored as ++ ; Supplemental Figure 2). The comparison of lung histopathlogical scores of 2 groups showed significantly enhanced lung injury in the ADS-MVA-vaccinated group at both 7 and 35 dpi compared with the control ADC-MVA group, suggesting that S-specific — but not MVA-specific - immunity promotes ALI during SARS-CoV infection (Figure 1E). Moreover, there is a moderate correlation between lung pathological scores and sera NAb titers at 0 dpi (Figure $1 \mathrm{~F}$ ), suggesting a role of S-specific antibody in enhancing SARS-CoV-mediated lung injury.

Anti-S-IgG induced severe lung injury during acute SARS-CoV infection. In our previous study, i.n. inocu-

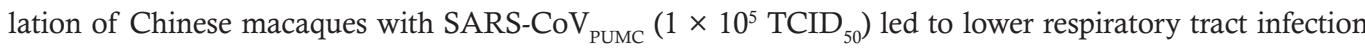
in all animals within 2 days (25). However, most of them rapidly cleared infection in the lungs, and all of the animals $(n=8)$ exhibited mild lung lesions before 3 dpi (25). Severe lung injury in SARS-CoV-infected Chinese macaques has not been detected until 7 dpi $(26,27)$. To determine the effect of anti-S antibody on the extent of SARS-CoV-mediated lung injury, we adoptively transferred $5 \mathrm{mg}$ (low dose) and $200 \mathrm{mg}$ (high dose) purified anti-S-IgG from ADS-MVA-vaccinated but unchallenged macaques into 2 groups of unvaccinated macaques ( $n=6$ /group) via i.v. injection, and then i.n. challenged recipients with SARS-CoV $\mathrm{PUMC}_{\mathrm{P}}$ $\left(1 \times 10^{5} \mathrm{TCID}_{50}\right)$ (Figure 2A). As controls, another 2 macaques were administered $200 \mathrm{mg}$ of control IgG (C-IgG) derived from ADC-MVA-vaccinated macaques. We sacrificed half of the macaques in each group at 2 dpi to avoid potential disruption by virus infection-induced antibodies against nucleocapsid protein $(\mathrm{N})$ and other viral proteins - and prior to when induction of ALI was typically observed. The remaining half of the macaques in each group was sacrificed at $21 \mathrm{dpi}$ to evaluate long-term impact (Figure 2A). At day 2 , sera from macaques that received low- or high-dose S-IgG showed neutralizing activity, with $\mathrm{IC}_{50}$ values ranging from 10-260 and 1,000-10,000, corresponding to the dose of sera transfer; macaques that received C-IgG failed to show neutralizing activity (Figure 2B and Supplemental Table 1). While NAb titers in the sera of macaques in the high-dose group declined steadily between 2 and $21 \mathrm{dpi}$, macaques in the low-dose and control groups displayed increased NAb titers over time (Figure $2 \mathrm{~B}$ ), suggesting active viral replication. 
A<smiles>CCCCC</smiles>

Chinese rhesus

macaques, $n=19$

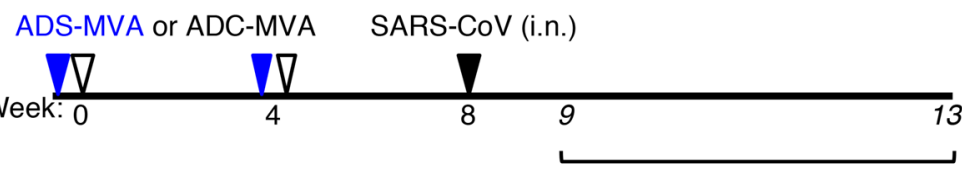

Sacrificed and tissue processing

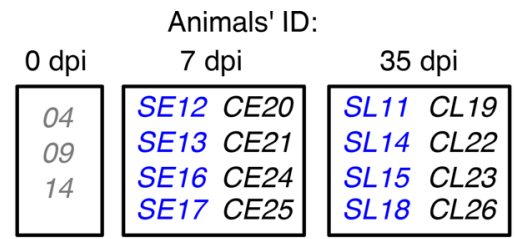

B

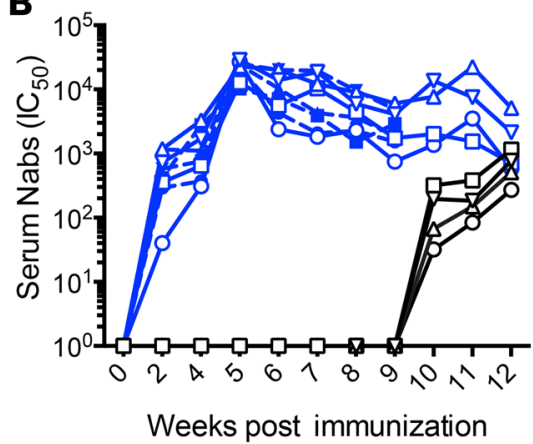

D

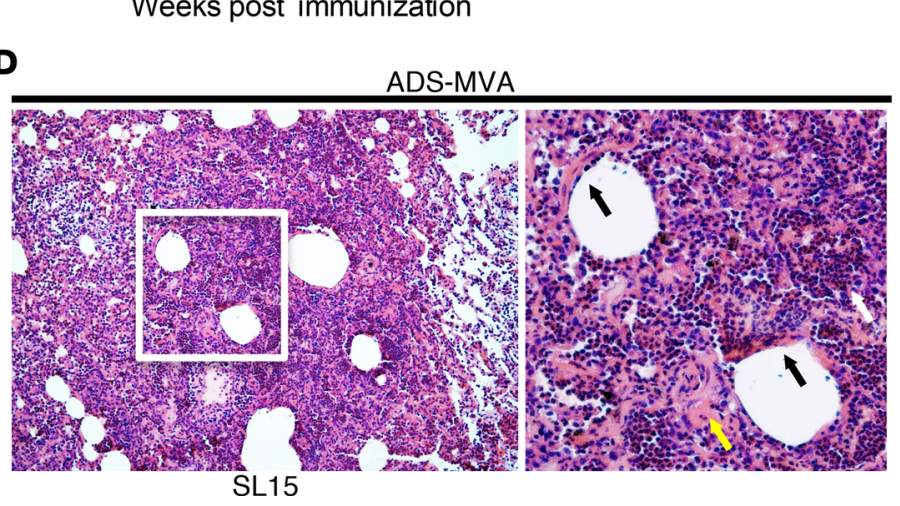

C

$$
\begin{aligned}
& \text { ADS-MVA ADC-MVA } \\
& \text { - SE12 — CE20 } \\
& \rightarrow \text { SE13 } \rightarrow \text { CE21 } \\
& \text { - SE16 \& CE24 } \\
& \rightarrow \text { SE17 } \rightarrow \text { CE25 } \\
& \text { - }- \text { SL11 - } 0 \text { CL19 }
\end{aligned}
$$

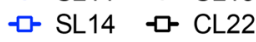

$$
\begin{aligned}
& \leadsto \text { SL15 } ₫ \text { CL23 } \\
& \rightarrow \text { SL18 } \rightarrow \text { CL26 }
\end{aligned}
$$

Weeks post immunization

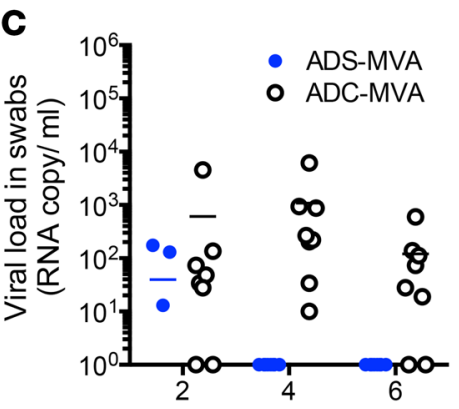

Days post infection

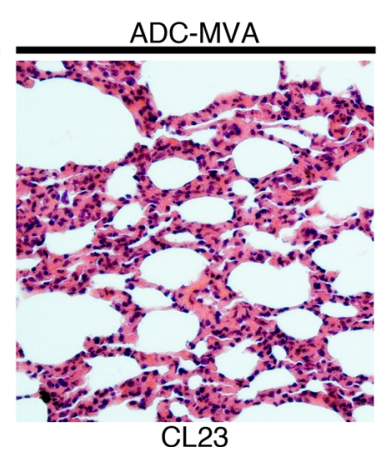

E

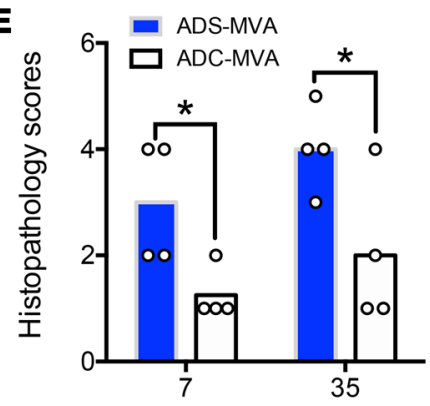

Days post infection

Figure 1. ADS-MVA-induced S-specific immune response enhanced pulmonary pathology in SARS-CoV-infected Chinese rhesus macaques. (A) Experimental design used to investigate the influence of S-specific immunity on SARS-CoV-induced lung injury. Two groups of Chinese rhesus macaques ( $n$ $=8$ /group) were subjected to i.m. injections of ADS-MVA or control vaccine ADC-MVA at weeks 0 and 4, followed by i.n. challenge with live pathogenic SARS-CoV ${ }_{\text {рuмc }}\left(1 \times 10^{5} \operatorname{TCID}_{50}\right)$ at 4 weeks after the second vaccination. Four animals each were sacrificed at 1 and 5 weeks after inoculation. Three healthy macaques were included as controls. (B) Serum neutralizing activity. Sera collected from macaques were tested for a capacity to neutralize SARS-CoV pseudotype virus. (C) Detection of viral RNA in oral swabs. SARS-CoV RNA was detected by nested RT-PCR in the swabs at the indicated time points relative to infection. (D) Pathology changes of the lung tissue. Sections were stained with H\&E. D shows symptom of acute DAD exhibited in 6 of 8 ADS-MVAvaccinated macaques with extensive exudation (yellow arrow), hyaline membranes lining the alveolar walls (black arrows), and massive cell infiltration in alveolar cavities (white arrow). Left image shows a low magnification overview (100x). Middle image shows higher magnification of the boxed area in left image (200x). Right image shows minor inflammation observed in 7 macaques received ADC-MVA $(n=8)$ with slight alveolar septa broadening and sparse monocyte infiltration (original magnification, 100x). (E) Histopathological scores of the ADS-MVA group, including lung samples collected at both 7 and 35 $\mathrm{dpi}$, were compared against the ADC-MVA control group. See Supplemental Figure 1 for the scoring index based on severity of lung histopathology. Data represent mean \pm SEM values. Statistical analysis was undertaken using 2 -tailed unpaired Student's $t$ test. ${ }^{*} P<0.05, n=4$. (F) Correlation of lung histopathological scores of all macaques with sera NAb titers at $0 \mathrm{dpi}$. Solid lines denote the relationship between histopathology scores and serum neutralizing activity. Statistical analysis was performed using Spearman's rank correlation test.

Indeed, SARS-CoV RNA was detected using real-time RT-PCR on oral swabs, and/or virus was recovered from lung tissue in both control animals and 5 of 6 animals (83\%) in the low-dose group. Only 2 of 6 animals $(33 \%)$ in the high-dose group were viral $\mathrm{RNA}^{+}$(Figure $2 \mathrm{C}$ and Supplemental Table 1), suggesting reduced viral production by high-dose S-IgG.

Consistent with the findings in the control ADC-MVA-vaccinated group, histopathological examination of the lungs indicated minor and moderate inflammation at day 2 and 21 in C-IgG recipients, respectively. In contrast, all S-IgG recipients exhibited symptoms of acute DAD with various degrees of exudation, hyaline membrane formation, and hemorrhage and inflammatory cells within alveolus at both day 2 and 21 (Figure 2, D and E, and Supplemental Table 2). Of note, despite the presence of high titer sera NAbs during the chronic stage of infection (Figure 1B and Figure 2B), only 1 of 5 C-IgG and control 
A

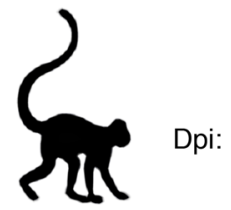

Chinese rhesus macaques, $n=14$

$\lg G$

SARS-CoV (i.n.)

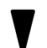

0

Sacrificed and tissue processing

\begin{tabular}{rcccccr}
\multicolumn{2}{c}{ Group ID: High dose } & Low dose & C-lgG & High dose & Low dose & C-lgG \\
Animals' ID: & HSO1 & LS07 & C13 & HSO4 & LS10 & C14 \\
& HS02 & LS08 & & HS05 & LS11 & \\
HS03 & LS09 & & HS06 & LS12
\end{tabular}
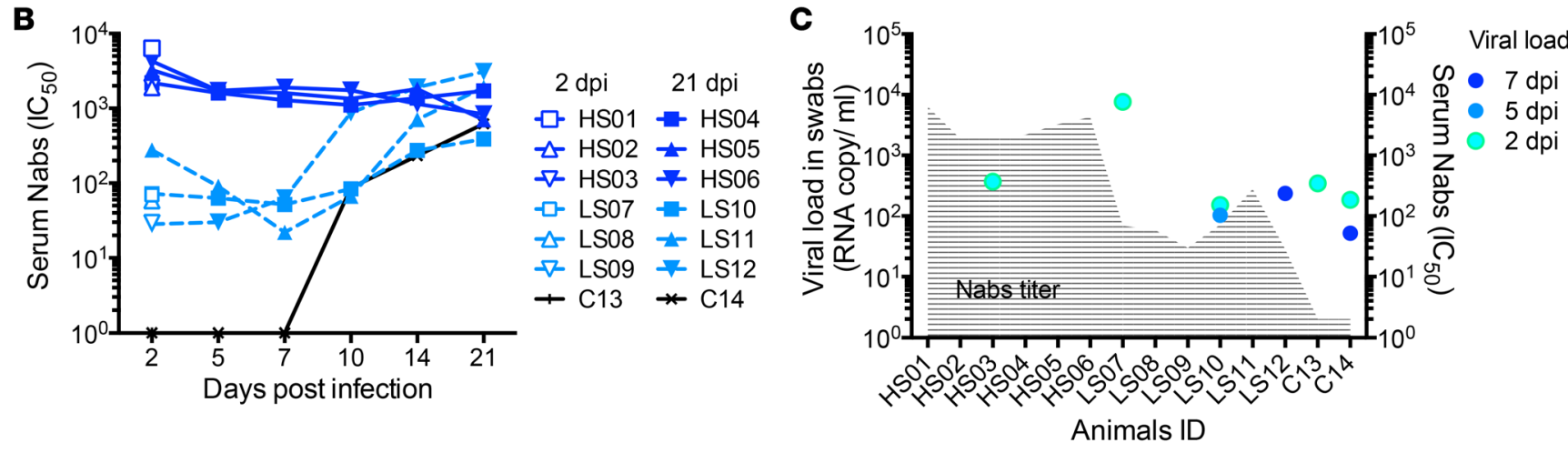

D

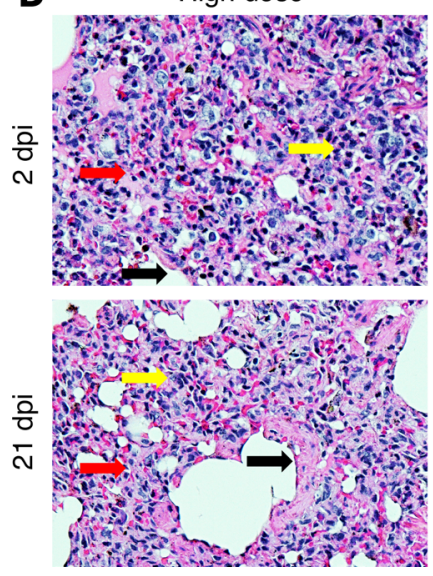

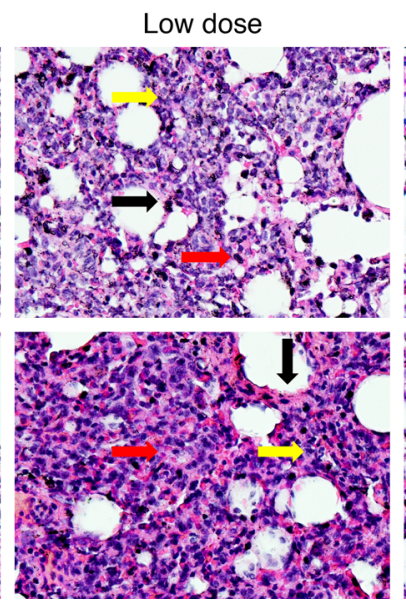

Low dose

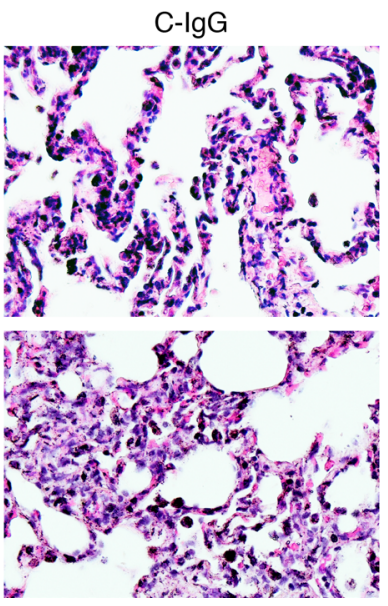

E

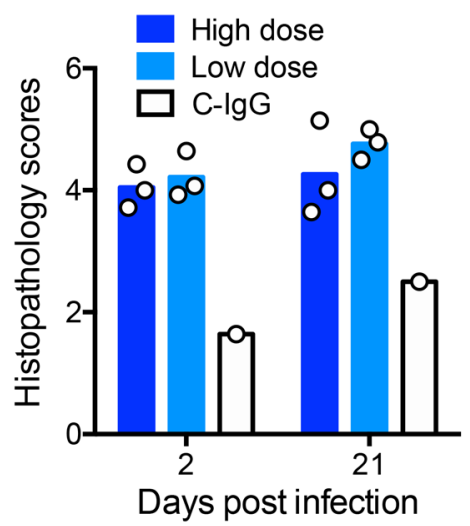

Figure 2. Anti-spike antibodies induced ALI in SARS-CoV-infected Chinese rhesus macaques. (A) Experimental design used to investigate the influence of S-IgG on SARS-CoV-induced lung injury. Two groups of Chinese rhesus macaques ( $n=6 /$ group) were subjected to i.v. injection of high-dose $(200 \mathrm{mg}$ ) or low-dose $(5 \mathrm{mg})$ purified S-IgG from ADS-MVA-vaccinated but unchallenged macaques. As controls, another 2 macaques were administered $200 \mathrm{mg}$ of C-IgC derived from ADC-MVA-vaccinated macaques. After 2 days, 3 groups of macaques were challenged i.n. with SARS-CoV $\mathrm{pumc}(1 \times$ $10^{5} \mathrm{TCI}_{50}$ ). Half of the animals from each group were sacrificed at 2 and $21 \mathrm{dpi}$. (B) Sera from macaques at the indicated time points were tested for the capacity to neutralize SARS-CoV pseudotype virus. (C) Detection of viral RNA in oral swabs. SARS-CoV RNA was detected by nested RT-PCR in the swabs from one of the high-dose S-IgG-treated macaques $(n=6), 3$ low-dose S-IgG-treated macaques $(n=6)$, and 2 C-lgG-treated macaques $(n=2)$ at the indicated time points. Left $y$ axis shows the viral RNA copy number per milliliter swab. Right $y$ axis shows the serum NAb titers of each macaque at $2 \mathrm{dpi}$, which are highlighted by shaded area. (D) Pathology changes of the lung tissue (200x). Sections from macaques were stained with H\&E. Images show symptom of acute DAD exhibited in macaques received high-dose and low-dose S-lgG $(n=12)$ with extensive exudation (red arrows), hyaline membranes (black arrows), and massive cell infiltration (yellow arrows) at 2 and $21 \mathrm{dpi}$. Right panel shows minor and moderate inflammation in the macaques received C-IgG with slight alveolar septa broadening and sparse monocyte infiltration at 2 and 21 dpi. (E) Histopathological scores of the high-dose and low-dose S-IgG groups, including lung samples collected at both 2 and 21 dpi, were compared against the C-IgG group. See Supplemental Figure 1 for the scoring index based on severity of lung histopathology.

ADC-MVA vaccine recipients showed acute DAD (Figure 1E and Figure 2E), indicating a time-restricted role for S-IgG, which mostly causes severe ALI in acutely infected macaques. Therefore, we conclude that, despite viral suppression, the presence of S-IgG at the acute stage of SARS-CoV infection caused severe ALI that persists until late stages. 
S-IgG failed to prevent SARS-CoV lower respiratory tract infection and amplified IMM infiltration and accumulation in the lungs. To determine the potential cause of S-IgG-enhanced ALI, we measured viral infection and monocytes/macrophages infiltration in the lungs and serum cytokine profile at 2 dpi. We previously found that pulmonary infection of Chinese macaques could be rapidly controlled. Viral $\mathrm{RNA}^{+}$cells were detectable in the lungs of only 2 of 4 animals at 2 dpi, although all of them were viral nucleoprotein-positive $\left(\mathrm{NP}^{+}\right)$in the lungs and the hilar lymph nodes (LNs), where the lymphatics of the lungs drain (25). Therefore, we measured viral $\mathrm{RNA}^{+}$cells by in situ hybridization (ISH), as well as NP signals in the lungs and the hilar LNs by IHC staining to evaluate the actual pulmonary infection of each macaque. Consistent with the results by viral isolation (Supplemental Table 1), viral RNA was detected in pneumocytes of 1 macaque in the high-dose (HS02, 33\%), 2 macaques in the low-dose (LS01, LS02; 66\%), and the C-IgG-treated macaque (Figure 3A and Supplemental Table 3), suggesting reduced productive infection by S-IgG. However, an NP antibody identified positive signals in the lungs and hilar LNs of all S-IgG and C-IgG recipients ( 7 of 7 macaques) but not healthy controls (Figure 3B and Supplemental Table 3), suggesting that S-IgG failed to prevent SARS-CoV entry, and SARS-CoV established lower respiratory tract infection in all of the challenged animals.

Consistent with previous findings in SARS-CoV-inoculated naive Chinese macaques $(n=4)(25)$, double-immunostaining with the NP antibody and antibodies specific to the stages of macrophage differentiation and inflammation (MAC387, CD68, CD163) revealed significant infiltration of IMMs in the lungs of C-IgG recipients, consisting of newly infiltrated $\left(\mathrm{MAC} 387^{+}\right)$and inflammatory $\left(\mathrm{CD} 163^{+}\right)$monocytes/ macrophages (Figure 3C and Supplemental Figure 3). The CD163+ cells greatly exceeded the CD68 ${ }^{+}$population and typically gathered around $\mathrm{NP}^{+}$cells to form cell clusters (Figure 3C), showing an association between viral infection and IMM infiltration in these macaques.

Compared with C-IgG recipients, more robust signals for MAC387 and CD163 were identified in the lungs of S-IgG recipients (Figure 3C and Supplemental Figure 3), suggesting enhanced IMM infiltration by S-IgG. MAC $387^{+}$and $\mathrm{CD} 163^{+}$monocytes/macrophages not only gathered around $\mathrm{NP}^{+}$cells, but also formed clusters in tissues with little NP signal (Figure 3C), suggesting that infection-induced recruitment from circulation continued after viral clearance and accumulated at the inflamed site. Moreover, more-concentrated $\mathrm{CD} 68^{+}$signals were found, indicating altered macrophages functional response by S-IgG treatment (Figure 3C). Consistently, elevated serum levels of IL- 8 were observed in macaques treated with high-dose S-IgG at 2 dpi (Supplemental Figure 3), and the IL-8 levels are strongly correlated with anti-SARS-CoV NAb titers (Supplemental Figure 3). IL-8 is a macrophage-derived inflammatory cytokine important in initiating ALI. These results, therefore, suggest that S-IgG likely altered macrophage functional response in the lungs during acute infection, resulted in increase in IL-8 production, and enhanced ALI and IMM infiltration and accumulation.

Alveolar monocytes/macrophages assumed a wound-healing function as early as 2 dpi in macaques not treated with S-IgG. Macrophage activation is dynamic and plastic. Different monocyte/macrophage activation statues have different specialized and critically timed roles, taking part in either the initiation and maintenance or resolution of inflammation (9). A well-organized wound-healing response leads to timely resolution of inflammation, whereas disturbance in wound-healing response results in persistent inflammation and injury. To understand the S-IgG-caused excessive inflammation, we further defined macrophage heterogeneity and activation statues in the lung lesions of C-IgG $(n=1)$ and S-IgG recipients $(n=3)$ at 2 dpi. Three healthy macaques and 4 naive Chinese macaques challenged with the same dose of SARS-CoV ${ }_{\text {PUMC }}$ and scarified at 2 dpi from a previous study were included as controls (25).

By combining 5 macrophages markers (MAC387, CD68, CD163, HAM56, and CD206) (28-30), 2 subpopulations of resident macrophages were shown in the lungs of Chinese rhesus macaques at steady state as previously described (29). They are interstitial macrophages (IMs; MAC $387^{+}{ }^{-}$AM56-CD206-) (Figure 4A) and resident alveolar macrophages (AMs; CD68 ${ }^{+}$HAM56-) expressing low levels of CD163 and CD206, a marker specific to the macrophage wound-healing response, corresponding to their intrinsic antiinflammatory function (Figure 4B) (29). In lung lesions of both C-IgG-treated and naive macaque

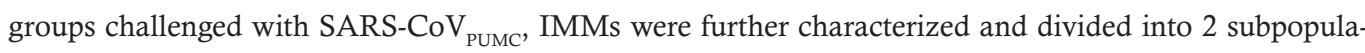
tions. One subpopulation was the recently recruited MAC387 ${ }^{+}$HAM56-CD68- monocytes (Figure 4C) (29), which is like IM but is larger in size and primarily coexpressed CD163 (Supplemental Figure 4). The other subpopulation was single $\mathrm{CD} 163^{+}$inflammatory macrophages that had differentiated from newly recruited MAC $387^{+}$monocytes/macrophages (Figure 4D and Supplemental Figure 4) (29). Importantly, resident 
AMs $\left(\mathrm{CD} 68^{+}\right)$differentiated into HAM56 ${ }^{+}$cells, with a slight increase in numbers and increased size, with stronger CD163 and - more importantly - CD206 staining, suggesting that they are alternatively activated (Figure 4D). Strikingly, elevated levels of TGF- $\beta$ expression were detected in all MAC387 ${ }^{+}$— and most enlarged $\mathrm{CD}_{163}{ }^{+}$- as well as $\mathrm{CD}^{+} 8^{+}$macrophages in the lungs of both C-IgG-treated and unvaccinated macaques compared with healthy controls (Figure 4, G-I), indicating that many IMMs and activated resident AMs have assumed a wound-healing function in infected lungs within 2 days of infection.

$S$-IgG treatment skewed wound-healing macrophage response in the lungs during acute SARS-CoV infection. Compared with the macaques not treated with S-IgG, the number of IMMs (MAC387 ${ }^{+}$and CD163 ${ }^{+}$HAM56 ${ }^{-}$) significantly increased in S-IgG recipients (Figure 4, E and F). Moreover, many recently recruited MAC387 ${ }^{+}$HAM56- monocytes/macrophages did not coexpress CD163 (Supplemental Figure 4), representing the newest recruited blood monocytes, as a result of enhanced monocytes infiltration by S-IgG. Importantly, the majority of CD163 ${ }^{+}$HAM56- IMMs coexpressed CD68 (Supplemental Figure 4), suggesting a change of function of this subpopulation by S-IgG. Moreover, resident macrophages $\left(\mathrm{CD}^{+} 8^{+} \mathrm{HAM} 56^{+}\right)$in the lungs of S-IgG recipients no longer express CD206, which — being surrounded by many CD163+ IMMs (Figure $4 \mathrm{~F}$ ) - suggests a loss of wound-healing function and a role in recruiting blood monocytes. Consistently, S-IgG-treated macaques displayed a loss of TGF- $\beta$ and high expression of IL- 6 in all macrophage subpopulations (Figure 4, G, H, and J). IL-6 favors macrophage accumulation the site of injury and is important for the development of persistent inflammation and ALI $(31,32)$. Therefore, we conclude that, while AMs underwent phenotypic and functional changes in acutely infected lungs, many monocytes/macrophages assumed a wound-healing function for inflammation resolution, but the presence of S-IgG skewed the wound-healing response, leading to uncontrolled inflammation and tissue injury.

Onset of an antibody response prior to viral clearance is associated with abrogated wound healing responses and increased IMM lung infiltration in unvaccinated Chinese rhesus macaques. To understand the mechanism underlying the time-restricted role of S-IgG in promoting ALI, we conducted a temporal analysis of the productive viral infection, antibody response, and macrophage functional changes in unvaccinated Chinese macaques during the first week of infection. Naive macaques were challenged i.n. with $1 \times 10^{5} \mathrm{TCID}_{50}$ SARS-CoV ${ }_{\text {PUMC }}$ and sacrificed at 2, 3, and 7 dpi (4 macaques/group) (Figure 5A) (25). Successful lower respiratory tract infection was confirmed by detection of viral NP using IHC staining in the lungs of all macaques (25). Viral $\mathrm{RNA}^{+}$alveolar pneumocytes were primarily found at 2 dpi ( 2 of 4 macaques), and infrequently at 3 dpi (0 of 4 macaques; Figure 5B and Supplemental Table 4) (25). At 7 dpi, viral RNA was detectable in the lungs of 2 macaques, AD0516 and AD0518, suggesting productive infection there (Figure $5, \mathrm{~B}$ and C). Only few scattered $\mathrm{NP}^{+}$cells were found in AD0517 (Figure 5D). Neither NP nor viral RNA was found in the lungs or swabs of AD0515, suggesting clearance of productive viral pulmonary infection in this macaque (Figure 5D and Supplemental Table 4). Serum NAb was not observed at 2 and 3 dpi until 7 dpi in 2 of 4 macaques, AD0515 and AD0516 (Supplemental Table 4 and Figure 5E).

In agreement with the findings in the C-IgG group, temporal analysis of macrophage heterogeneity revealed significantly increased number of IMMs $\left(\mathrm{CD} 163^{+} \mathrm{CD} 206^{-}\right)$in viral $\mathrm{RNA}^{+}$lungs at 2 dpi (Figure 5F, right image). The IMMs diminished at 3 dpi (4 of 4 macaques) (Figure 5G) and became nearly completely absent at $7 \mathrm{dpi}$ in macaques without productive pulmonary viral infection ( 2 of 4 macaques, AD0515 and AD0517) (Figure 5H), indicating that inflammatory response resolved with 7 days following the control of productive infection. Critically, wound-healing macrophages $\left(\mathrm{CD} 163^{+} \mathrm{CD} 206^{+}\right.$and $\left.\mathrm{CD}_{163^{+}} \mathrm{TGF}-\beta^{+}\right)$were observed in the lungs of all macaques at $2 \mathrm{dpi}$, despite productive pulmonary viral infection in macaques AD0506 and AD0508 (Figure 5F). In contrast, macaque AD0516, with serum NAbs and productive virus infection in the lungs, displayed a significantly enhanced proinflammatory macrophage response and reduced inflammatory-resolving macrophages (Figure 5I), which was not observed in macaques with productive viral infection but undetectable NAb responses (AD0518) or in macaques with detectable NAbs but undetectable viral replication (AD0515) (Figure 5D and Supplemental Table 4). Therefore, persistent viral replication and concomitant NAb detection is associated with increased IMM lung infiltration and reduced wound-healing macrophage numbers.

$S$-IgG modifies functional response of alternatively but not classically activated macrophages to SARS-CoV infection. To determine whether S-IgG skews wound-healing response through modifying wound-healing macrophage functional response during SARS-CoV infection, we treated in vitro-polarized alternatively activated human monocyte-derived macrophages (MDM) for 20 hours with SARS-CoV pseudovirus and heat-inactivated sera of high-dose S-IgG-treated macaques (S-IgG sera) collected at 2 dpi. We 
A

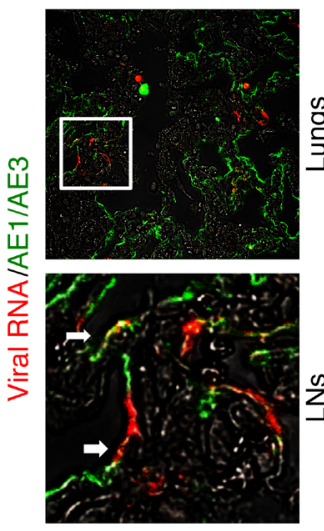

B

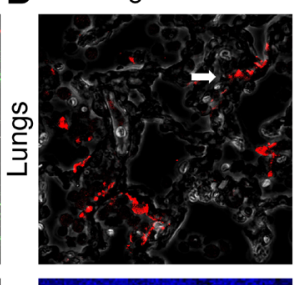

Low dose
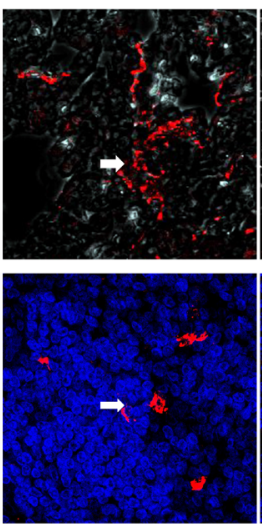

C-IgG

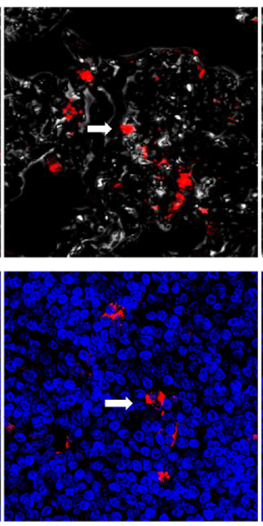

Healthy control
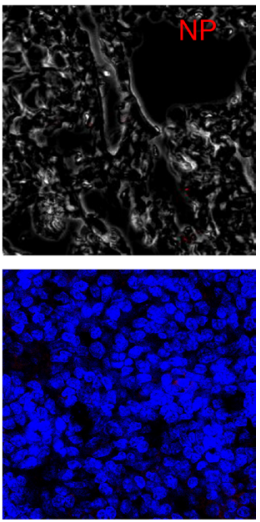

C
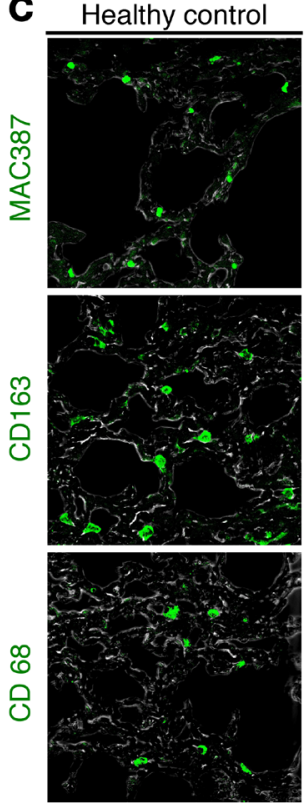

Bright field

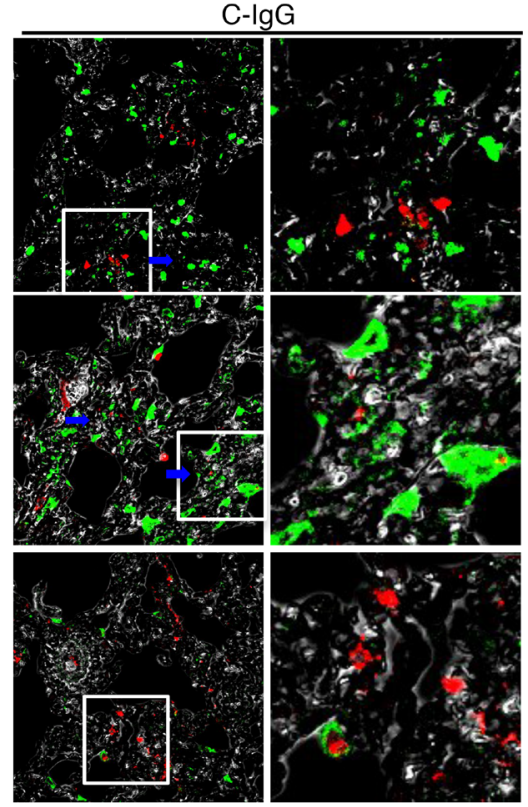

NP/Bright field

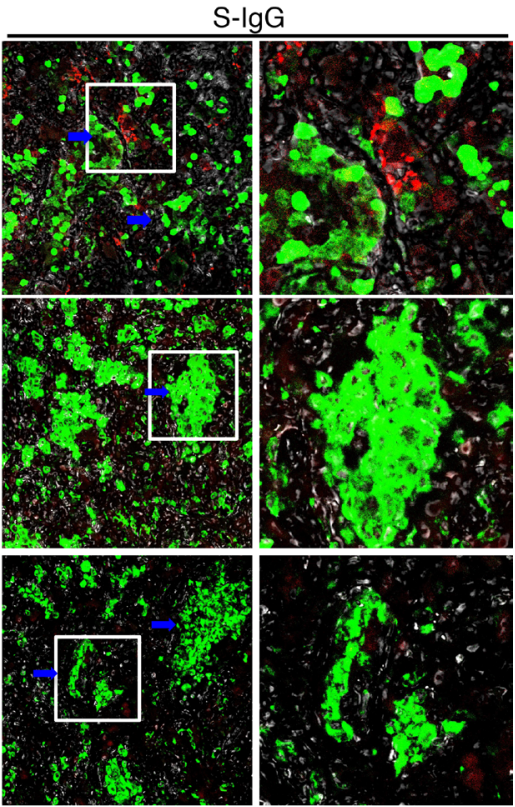

NP/Bright field

Figure 3. SARS-CoV infection and monocytes/macrophages infiltration in the lungs of C-IgG- and S-lgG-treated macaques at $\mathbf{2}$ dpi. (A) Representative images of SARS-CoV RNA+ (TRITC) and AE1/AE3+ (FITC) cells in the lungs of infected macaques (white arrows). The upper photo shows a low magnification overview (200x); the bottom photo shows the boxed area in the upper photo. (B) Representative images of viral protein (NP) immunostaining of the lungs and hilar lymph nodes (LN) of 7 infected and 3 uninfected animals (TRITC, white arrows) (original magnification, 200x). (C) Representative images of viral NP and monocytes/macrophages. These sections showed significantly increased IMMs in the lungs of the S-lgC group compared with the C-IgC group (MAC387', CD163+, or CD68 ${ }^{+}$; blue arrows). Tissue samples are double-immunostained for the SARS-CoV NP (TRITC) and markers for macrophages, including MAC387 (FITC), CD163 (FITC), and CD68 (FITC). Within the panel of representative images for the C-IgC and S-IgC groups, the left panel shows a low magnification overview; the right panel shows the boxed area in the left panel (original magnification, 200x).

then measured the concentrations of 13 inflammatory cytokines in cell culture supernatants, including IL-1 $\beta$, IFN- $\alpha$, IFN- $\gamma$, TNF- $\alpha$, MCP1, IL-6, IL-8, IL-10, IL-12p70, IL-17A, IL-18, IL-23, and IL-33. Sera from C-IgG recipients or healthy macaques were included as controls. In vitro-polarized classically activated MDM was also studied and compared with alternatively activated MDM in parallel. Consistent with previous report, unstimulated classically activated MDM produced high levels of IL-8 and IL-6; alternatively activated MDM only produced minimal levels of those cytokines (Figure 6A). Addition of SARS-CoV pseudovirus induced IL- 8 production in alternatively activated MDM to levels comparable with those found in unstimulated, classically activated MDM, as well as in low-level MCP1 and IL-6 production (Figure 6B). By contrast, SARS-CoV pseudovirus caused less than a 1-fold increase in IL-8 secretion, but not other cytokine secretion, by classically activated MDM (Figure 6C). Strikingly, while none of the sera samples induced cytokine production when they were administered into MDMs alone, 

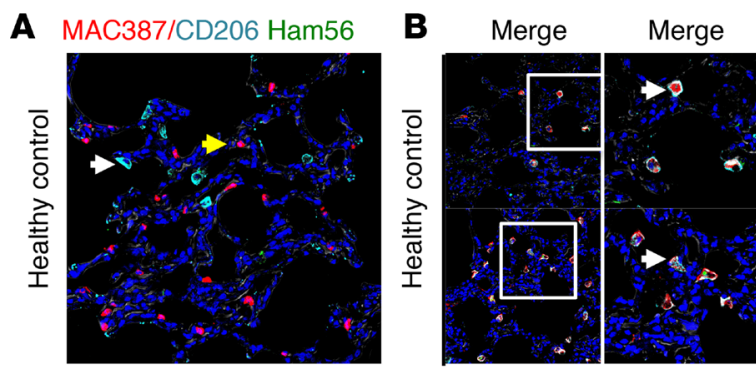

CD206 CD68/CD163 Ham56
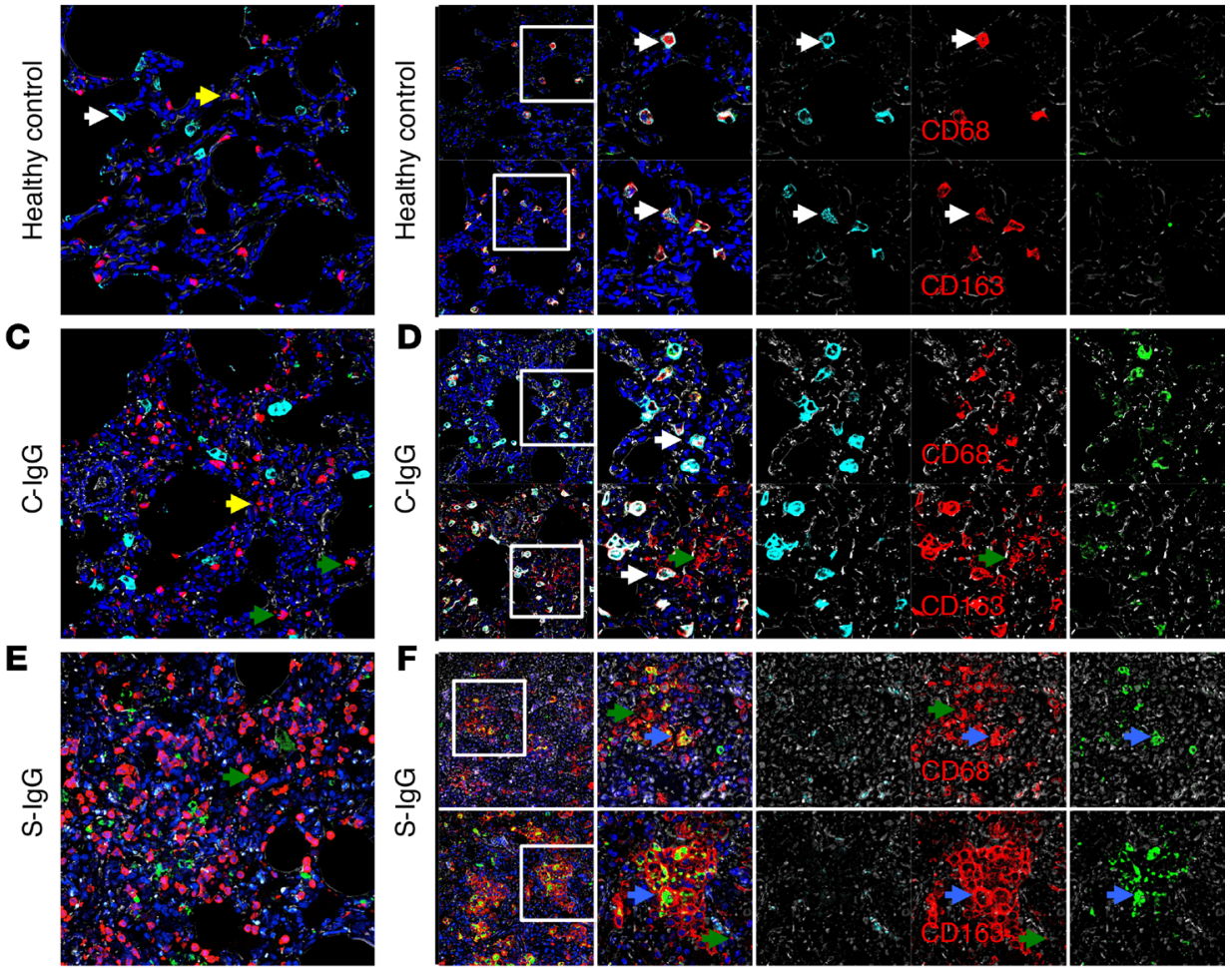

F

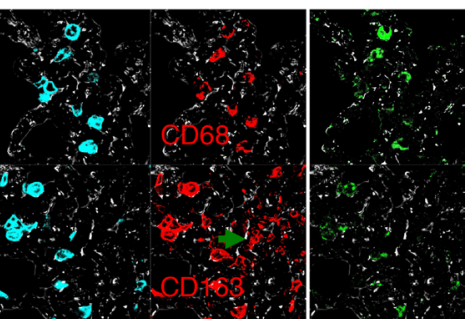

G

TGF $\beta$
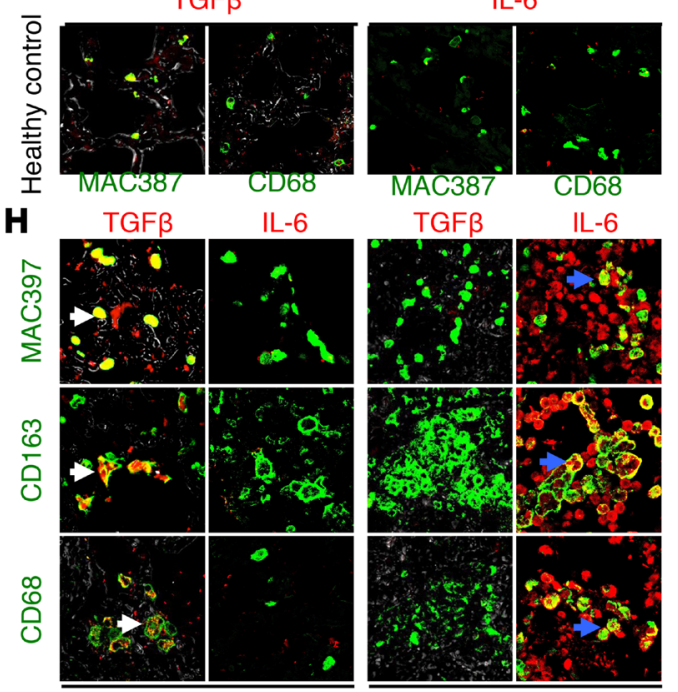

C-lgG
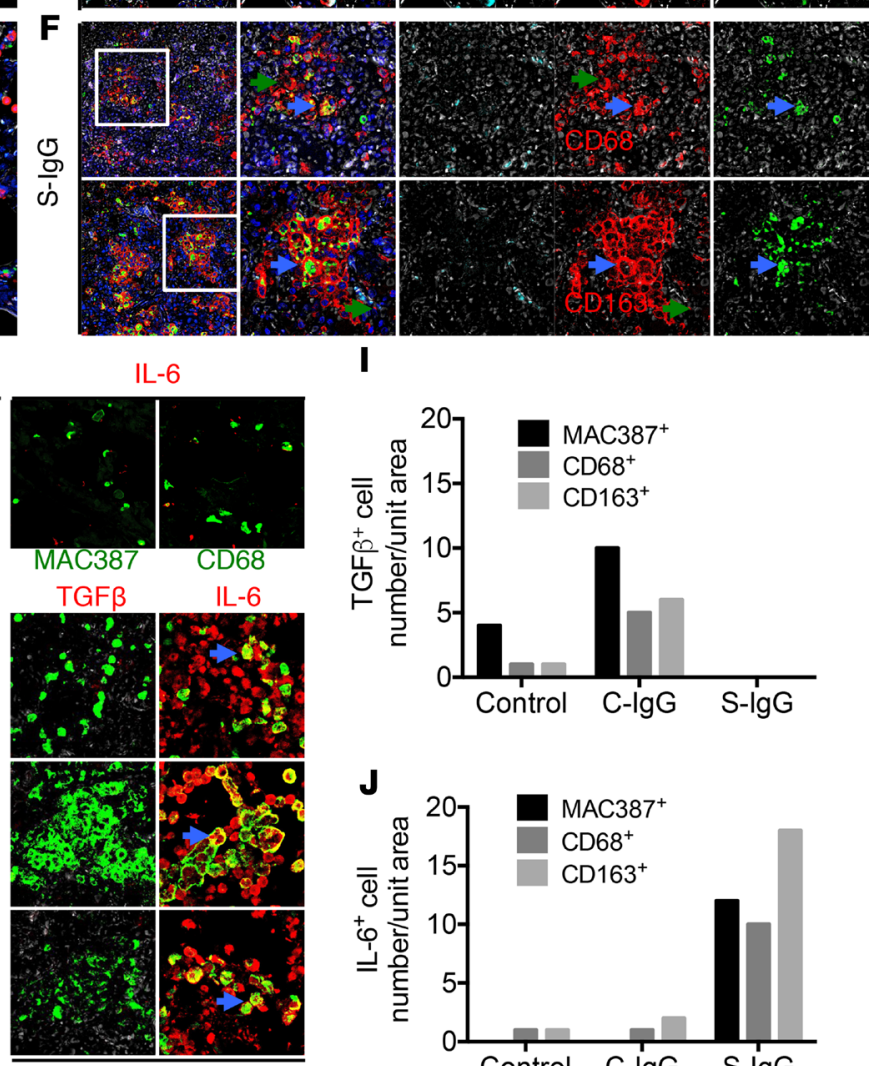

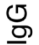

IL-6

I
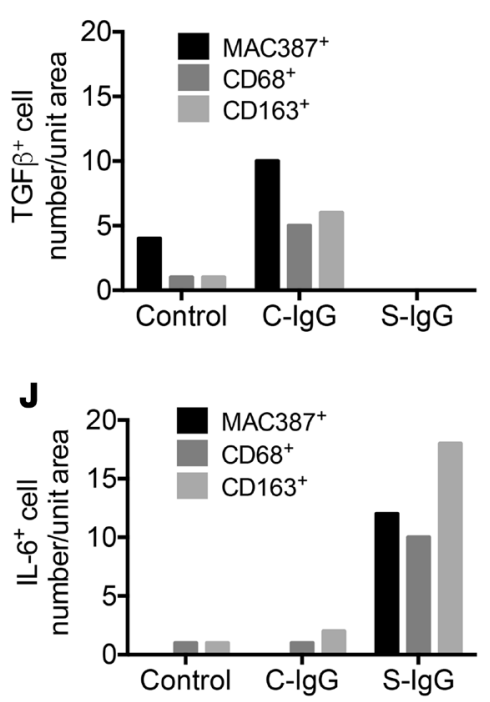

Figure 4. Comparison of monocyte/macrophage phenotype and function in the lungs of S-IgG- and C-IgG-treated macaques. (A-F) Phenotype of monocytes/macrophages subpopulations in the lungs of healthy, C-IgG-, and S-IgG-treated macaques (original magnification, 200x). These sections were triple-immunostained with antibodies for CD206 (cyan), HAM56 (FITC), and MAC387 (TRITC) (A, C, and E); CD206 (cyan), HAM56 (FITC) (B, D, and F), and CD68 (TRITC) (upper panel in B, D, and F); or CD163 (TRITC) (lower panel in B, D, and F). A and B show 2 subpopulations at steady states, includ-

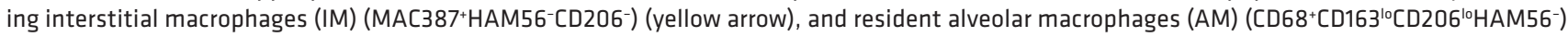
(white arrows). $\mathbf{C}$ and $\mathbf{D}$ show the presence of alternatively activated resident AM and accumulated IMMs in the C-IgC group. Alternatively activated

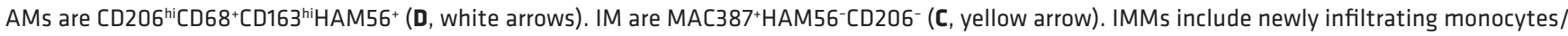
macrophages (MAC387+) (C, green arrow); and inflammatory macrophages (CD163+CD68-CD206-HAM56-) (D, green arrow). E and $\mathbf{F}$ show significantly increased number of IMMs and decreased number of alternatively activated macrophages in the S-IgG group. IMMs include newly infiltrating monocytes/macrophages $\left(\mathrm{MAC387}^{+}\right)\left(\mathbf{E}\right.$, green arrow) and inflammatory macrophages that are $\mathrm{CD}^{2} 8^{+} \mathrm{HAM}^{-} 6^{-}$(green arrow, $\mathbf{F}$, upper panel) and CD163 ${ }^{+}$ CD206-HAM56- (green arrow, F, lower panel). Resident AMs are no longer expressing CD206 (CD68+HAM56+CD163+CD206-) (blue arrow, F, upper panel). (G-J) TCF- $\beta$ and IL-6 expression in macrophages in the lungs. These sections were double-immunostained with antibodies for TGF- $\beta$ or IL- 6 (TRITC) and MAC387, CD163, or CD68 (FITC). G shows low expression levels of TCF- $\beta$ and IL- 6 in IMs (MAC387 ${ }^{+}$) and AMs (CD68 ${ }^{+}$) in healthy macaques. H shows increased TCF- $\beta$ expression in the C-IgC group (white arrows) but increased IL-6 in the S-IgC group (blue arrows). I and J show the numbers of TCF- $\beta^{+}$ and IL-6+ monocytes/macrophages in 3 groups in a $200 \times$ field. 
A

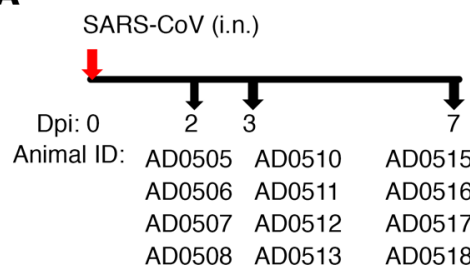

B

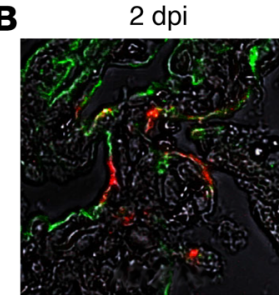

AE1/AE3 Viral RNA

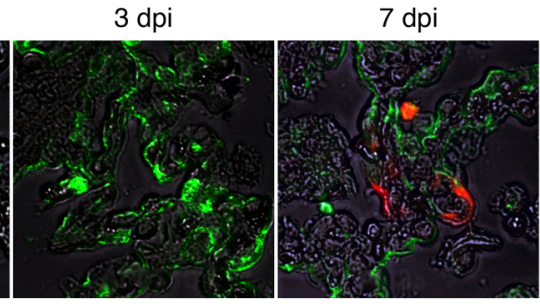

C

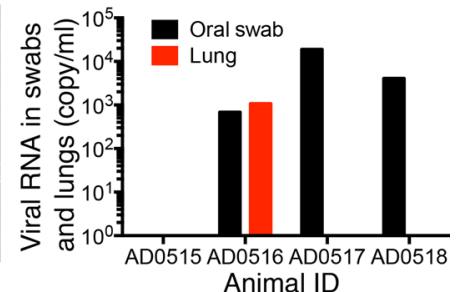

D

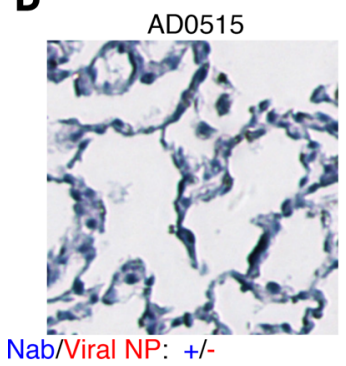

$\mathbf{F}$

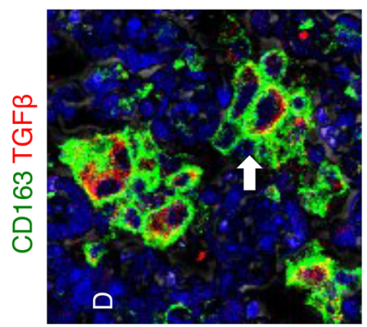

Nabs - / viral RNA+

$2 \mathrm{dpi}$
AD0516

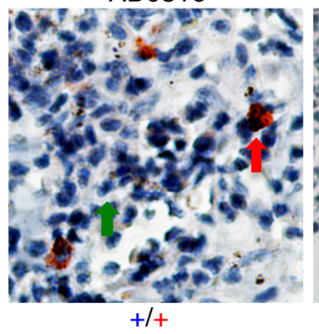

AD0517

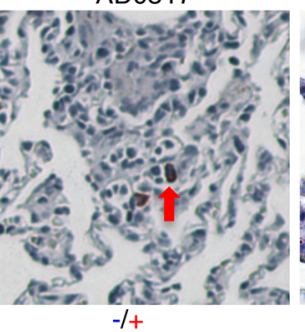

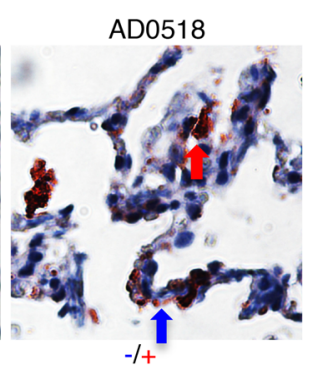

E

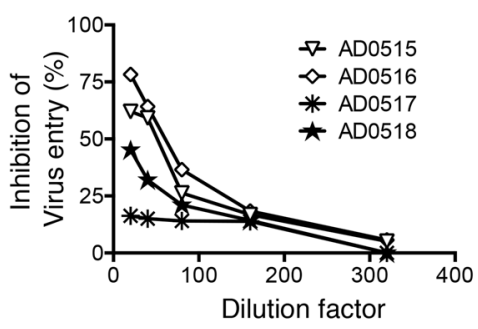

G

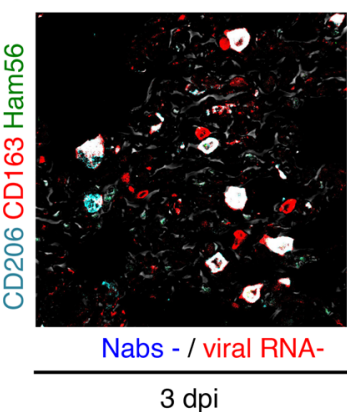

H

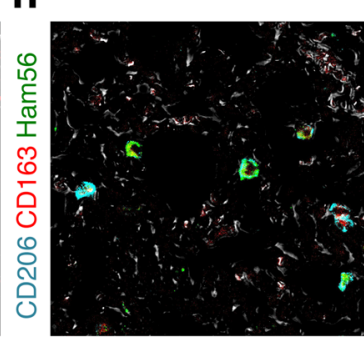

Nabs +/ viral RNA-
I

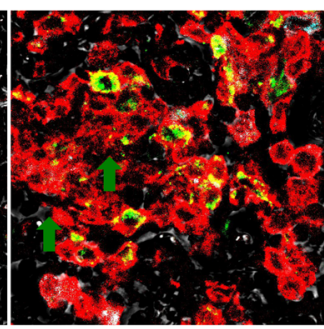

$7 \mathrm{dpi}$

Figure 5. Dynamics of the viral replication, antibody response, and macrophage activation during acute SARS-CoV infection. (A) Experimental scheme. A total of 12 Chinese rhesus macaques were challenged i.n. with SARS- $\operatorname{CoV}_{\text {pumc }}\left(1 \times 10^{5} \operatorname{TCID}_{50}\right)$. Four animals each were sacrificed at 2 , 3 , and $7 \mathrm{dpi}$. (B) SARS-CoV RNA detection by ISH in the lungs at 2, 3, and $7 \mathrm{dpi}$ (original magnification, $200 \times$ ). Viral RNA (TRITC) type I pneumocytes (FITC) was found at $2 \mathrm{dpi}(n=2)$ and $7 \mathrm{dpi}(n=1)$. (C) Viral RNA in the swabs and lung homogenates on $7 \mathrm{dpi}$. SARS-CoV RNA was detected using nested RT-PCR in swabs from 3 macaques (AD0516, AD0517, and AD0518) and lung homogenates from macaque AD0516. (D) Viral antigen and inflammatory infiltrates in the lungs at $7 \mathrm{dpi}(200 \times)$. These sections were stained for SARS-CoV NP (red) and nucleus (blue) by IHC, showing NP signal in macrophage-like cells in AD0516 and AD0517 (red arrows), and type I pneumocytes in AD0518 (blue arrows), but substantial inflammatory infiltrates were only observed in AD0516 (green arrows). (E) Serum neutralizing activity. Serum neutralizing activity was detected in macaques AD0515 and AD0516 at $7 \mathrm{dpi}$. (F-I) Decreased wound-healing response in macaques with productive pulmonary viral infection and serum neutralizing activity (200x). These sections were double-immunostained for TCF- $\beta$ (TRITC) and CD163 (FITC) or triple stained for CD206 (cyan), HAM56 (FITC), and CD163 (TRITC). The figure shows that wound-healing response took place within $2 \mathrm{dpi}$, with alternatively activated monocytes/macrophages (TCF- $\beta^{+}$and CD206 ${ }^{+}$, white arrows) and IMMs (CD163+CD206-, green arrows) coexisting in the lungs (F). After viral clearance, IMMs diminish at 3 dpi (G), and the homeostasis was restored at $7 \mathrm{dpi}(\mathrm{AD0515})$ (H). When pulmonary viral infection persists, IMM (CD163+CD206-' green arrows) infiltration and accumulation is enhanced, and wound-healing macrophages (CD206') are reduced in macaques that have faster NAb response (AD0516) (I).

administration of S-IgG sera caused a dose-dependent increase in the production of all 3 inflammatory cytokines in SARS-CoV-stimulated alternatively activated MDM, resulted in a greater than 10-fold increase in IL-6, an 8-fold increase in MCP1, and a 5-fold increase in IL-8 (Figure 6, D and E). This enhancement, however, was not observed when S-IgG sera were replaced with sera from C-IgG recipients or healthy macaques (Figure 6, F, G, and H), suggesting that S-IgG, but not other sera components, enhanced SARS-CoV-stimulated cytokine production by alternatively activated MDM. Administration of S-IgG sera had no effect on cytokine production by SARS-CoV-treated classically activated MDM (Figure 6, I, J, and K), indicating that S-IgG do not modulate virus-mediated classically activated macrophage functional response. MCP1 and IL-8 are key cytokines that regulate monocytes/macrophages migration and promote neutrophils infiltration, while IL- 6 promotes persistent inflammation and injury. These results suggest that, in response to SARS-CoV infection, wound-healing macrophages produce IL- 8 and low amounts of MCP1 to recruit neutrophils and blood monocytes, whereas the presence 

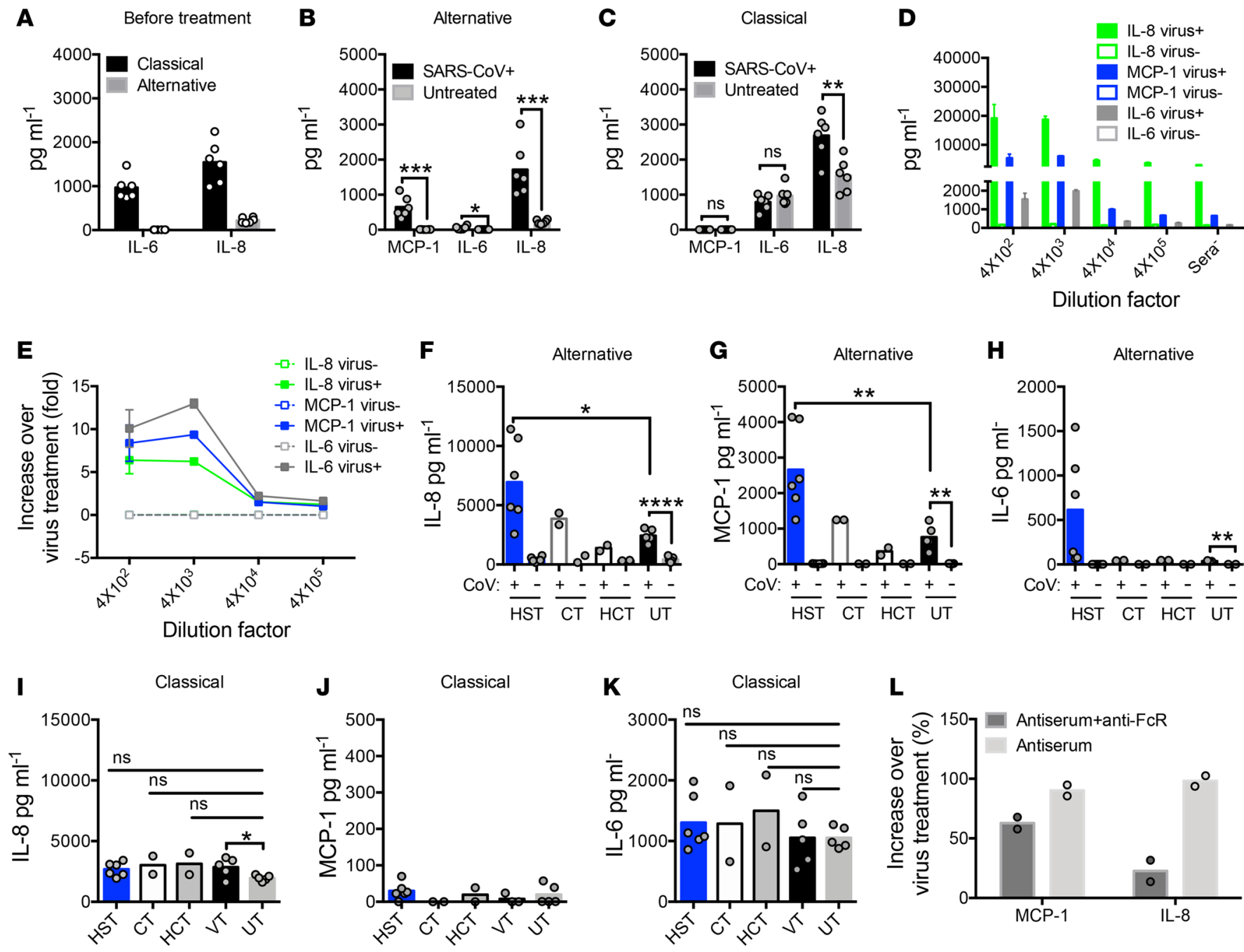

Figure 6. S-IgG significantly amplified proinflammatory cytokine production by SARS-CoV-treated alternatively activated macrophages. In vitropolarized alternatively activated macrophages were either left unstimulated or were incubated with SARS-CoV pseudovirus alone or cocultured with SARS-CoV pseudovirus and diluted sera from the high-dose S-IgG group $(n=6)$ or C-IgG group $(n=2)$ collected at 2 dpi or from healthy macaques ( $n$ $=2$ ) for 20 hours. Secreted cytokine and chemokine levels were measured in the culture supernatant and are shown in data represented as a column graph (A-D and F-K) and fold or percentage over supernatants from macrophages treated with virus alone (E and $\mathbf{L}$ ). A shows the levels of IL-8 and IL-6 in the supernatant of classically activated and alternatively activated MDM before treatment. B and C show that SARS-CoV treatment induced MCP1, IL-8, and very low levels of IL-6 production by alternatively activated MDM, as well as enhanced IL-8 production by classically activated MDM. D and $\mathbf{E}$ show that sera from S-IgG recipients caused dose-dependent increase in cytokine production from SARS-CoV-treated alternatively activated MDM. F-H show that virus treated alternatively activated MDM (VT), but not sera treated or untreated cells (UT), secreted proinflammatory cytokines IL-8 (F), MCP1 (G), and very low levels of IL-6 (H). Addition of sera (1:4,000 dilutions) from high-dose S-IgC (HST), but not C-IgC treated macaques (CT) or healthy controls (HCT), significantly amplified IL-8 (F), MCP1 (G), and IL-6 (H) production. I-K show that addition of sera (1:4,000 diluted) from high-dose S-IgG-treated (HST), C-IgG treated macaques (CT), or healthy controls (HCT) had no effect on IL-8 (I), MCP1 (J), and IL-6 (K) production by SARS-CoV-treated classically activated MDM. $\mathbf{L}$ shows that blockade of Fc $\gamma$ R significantly reduced antiserum-enhanced IL-8 secretion and partially reduced MCP1 secretion. Data represent mean values or mean values \pm SEM of at least 3 independent experiments. Two-tailed unpaired Student's $t$ test was used for statistical analysis. ${ }^{*} P<0.05$; ${ }^{*} P<0.01$; ${ }^{* *} P<0.001$; ${ }^{* * *} P<0.0001$.

of S-IgG can result in 5- to 10-fold increases in IL-8, MCP1, and IL-6 production by wound-healing macrophages, leading to significantly enhanced and persistent monocytes infiltration and tissue injury. Critically, in the presence of $\mathrm{Fc} \gamma$ receptor $(\mathrm{Fc} \gamma \mathrm{R})$ blocking antibody, IL-8 was significantly reduced when cells were treated with SARS-CoV pseudovirus and sera from high-dose S-IgG-treated macaques (diluted 1:4,000), indicating that anti-SARS-CoV antibody modifies wound-healing response partially through FcyRs (Figure 6L). Therefore, we conclude that S-IgG directly modifies functional response of alternatively activated macrophages during acute infection, thus skewing wound-healing response, leading to hypercytokinemia, aggressive inflammation and severe lung injury. 
Deceased SARS patients displayed higher levels of serum NAbs, accumulated proinflammatory, macrophages infiltration, and absence of wound-healing macrophages in the lungs. To examine the role of S-IgG in promoting ALI in a clinical setting, we investigated antibody responses during the early stage of infection in 2 groups of deceased patients or individuals recovered from SARS and characterized the heterogeneity of monocytes/ macrophages in the lungs of 3 deceased SARS patients. H\&E staining of lung specimens derived from 3 deceased SARS patients revealed severe ALI (Figure 7A). Consistent with the macaque findings, IHC analysis revealed significant infiltration of $\mathrm{CD} 163^{+}$monocytes/macrophages into the lungs of patients, with little TGF- $\beta$ expression and loss of the wound-healing marker CD206 ${ }^{+}$, indicating predominantly proinflammatory macrophage activation and reduced inflammatory-resolving response (Figure 7B). Moreover, significantly higher levels of anti-S NAbs were detected in the sera of deceased patients $(n=6)$ during acute infection compared with recovered patients $(n=8)$ (Figure 7C). These results, therefore, are consistent with findings in experimental macaques.

Antisera from deceased SARS patients skewed wound-healing response during SARS-CoV infection partially through the $F c \gamma$. To examine whether human anti-SARS-CoV antibody modifies wound-healing response during SARS-CoV infection, we treated in vitro-polarized alternatively activated MDM with SARS-CoV pseudovirus for 20 hours in the presence of sera collected from SARS patients during acute infection. Macrophages treated with SARS-CoV pseudovirus or sera alone were included as controls. We then examined the protein levels of cytokines/chemokines in the cell culture supernatant. Consistent with findings in macaques, administration of sera from a deceased SARS patient (D1; Supplemental Table 5) into SARS-CoV-treated alternatively activated MDM caused a dose-dependent increase in the production of cytokines, leading to a 2- to 3-fold increase in MCP1 and IL-8 after 20 hours (Figure 8, A and B). This enhancement, however, was not observed in cells treated with sera alone (Figure 8, A-D). Administration of sera from recovered patients ( $n=8$; Supplemental Table 5) had no effect on cytokine production except 1 sample (Figure 8, C and D). These differences may be explained, at least in part, by their lower NAb titers, although it is also possible that sera from recovered patients contain different antibody populations. Consistently, the only serum sample - R2, which enhanced chemokine production - has a relatively higher NAb titer among the samples tested (Supplemental Table 5). Moreover, statistical analysis shows that IL-8 production strongly correlates with NAb titer of sera from deceased patients (Figure 8E). Furthermore, IL-8 production was significantly reduced when cells were treated with SARS-CoV pseudovirus and sera from deceased SARS patient (diluted 1:4,000) in the presence of Fc $\gamma$ R blocking antibody, indicating that antiSARS-CoV antibody modifies wound-healing response in humans partially through Fc $\gamma$ Rs (Figure 8F).

\section{Discussion}

Here, we present evidence of a detrimental role of anti-S-IgG in ALI during SARS-CoV infection. Respiratory CoVs infection poses a unique challenge to the immune system: not only must the virus be rapidly eliminated, but lung inflammation must also be controlled to prevent acute respiratory failure (33). In the present study, we show that, despite markedly reducing virus titers, anti-S-IgG caused lung injury during the early stages of infection by abrogating a wound-healing macrophage response and TGF- $\beta$ production, while promoting proinflammatory cytokine IL- 8 and MCP1 production and inflammatory macrophage accumulation. To our knowledge, our data demonstrate a previously unrecognized mechanism underlying virus-mediated ALI and suggest that modulation of the anti-S antibody response or blockage of Fc $\gamma$ receptors during acute infection might be needed for effective treatment for respiratory $\mathrm{CoV}$ infection.

Many studies have demonstrated the role of NAbs induced by the S glycoproteins in protecting viral replication in susceptible hosts, including mice, ferrets, hamsters, and macaques $(24,34,35)$. However, the effect of S-specific immunity in protecting against pulmonary immunopathology has been controversial. In some cases, vaccination-induced immunity assists the viral clearance and protects mice or ferrets against lethal challenge (36-39), whereas in many other situations, multiple vaccine platforms appear to induce increased eosinophilic proinflammatory pulmonary response upon challenge $(18,23,40)$. These differences may be explained, in part, by the characteristics of the vaccine being studied, the infectious dose, and the viral strain employed, as well as by the type or quality of immune response elicited. Recent studies suggest that $\mathrm{CD}^{+} \mathrm{T}$ cell response plays a crucial role in viral clearance and protection of mice against eosinophilic immunopathology and lethal SARS-CoV infection, whereas immunopathology predominantly reflects an inadequate vaccine-induced $\mathrm{Th} 1$ response (20-23). 
A

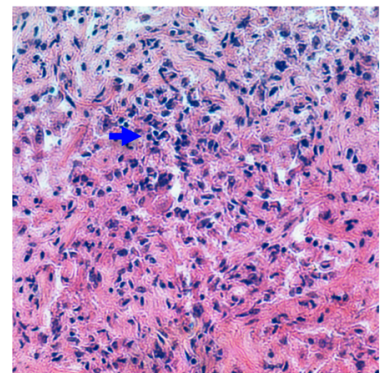

B CD163 Ham56 CD206

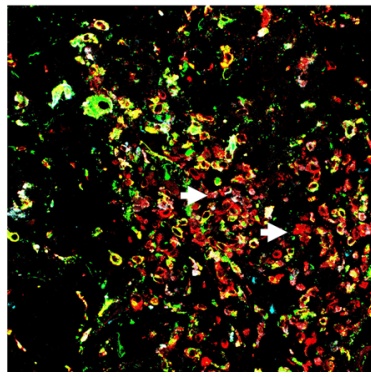

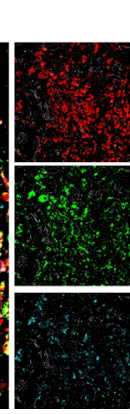

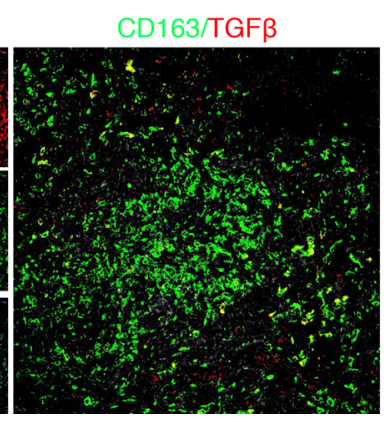

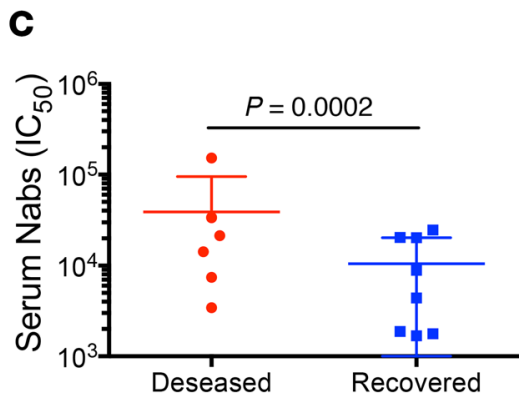

Figure 7. NAb response and phenotype analysis of accumulating monocytes/macrophages in the lungs in deceased SARS patients. (A) Pathology changes of the lung tissue. Sections from deceased SARS patients were stained with H\&E. A is representative of 3 patients (original magnification, $100 \times$ ), showing symptom of acute DAD with massive cell infiltration in alveolar cavities (blue arrow). (B)Massive accumulation of IMMs and absence of wound-healing macrophage response in the lungs of deceased patients. These sections were triple-immunostained with antibodies for CD206 (cyan), HAM56 (FITC), and CD163 (TRITC) or double-immunostained with TCF- $\beta$ (TRITC) and CD163 (FITC) (original magnification, 200x). The right panel in the left image shows single colors from the left image (200x). These representative images show accumulation of IMMs (CD163+CD68- ${ }^{-}$CD206- $^{-}$HAM56- $^{-}$ white arrows) and the absence of wound-healing response, indicated by loss of the signal for CD206 (cyan, B1) and TCF- $\beta$ (TRITC, B2) in the lungs. (C) Serum-neutralizing activity against SARS-CoV. Sera collected from deceased (red, $n=6$ ) or recovered (blue, $n=8$ ) SARS patients at the early stage of infection were tested for the capacity to neutralize SARS-CoV pseudotype virus. Two-tailed unpaired Student's $t$ test was used for statistical analysis.

In fact, a protective effect against pulmonary immunopathology mediated by full length $\mathrm{S}$ proteinbased vaccine in SARS-CoV-infected nonhuman primate models has not been described, to our knowledge. Although several vaccine candidates protected macaques against viral replication in the lungs, pulmonary immunopathology was not evaluated in these studies $(24,41,42)$. By contrast, it was shown that SARS-CoV-specific immune memory induced by prior infection enhanced lung inflammation following homologous challenge in African green monkeys (17). Similarly, augmentation of disease by vaccination has also been described in studies of atypical measles and dengue hemorrhagic fever, as well as several respiratory diseases, including respiratory syncytial virus (RSV) and pandemic influenza $(16,43)$. In the study of RSV vaccine conducted in 1966 and 1967, 80\% of RSV vaccinees needed hospitalization, whereas only $5 \%$ of RSV-infected children in the control vaccine group required admission, although the underlying mechanisms remain incompletely understood. Using Chinese rhesus macaques, we show that an ADS-MVA-based vaccine protected macaques against viral replication but significantly enhanced acute DAD at both 7 and 35 dpi compared with the control ADC-MVA group, suggesting that S-specific but not MVA-specific immunity promotes ALI (Figure 1). Until now, it remains unknown if SARS vaccine candidates with a focus on the receptor-binding domain (RBD) of $S$ protein can avoid the induction of ALI in nonhuman primates. These vaccines have been shown to induce highly potent NAb responses and elicit long-term protective immunity in immunized small animals (38).

SARS-CoV infection of Chinese macaques is often characterized by the rapid control of viral replication with mild lung lesions in most macaques (25). The mechanisms underlying why Chinese macaques do not often develop ALI, as observed in most SARS patients, are largely unknown. Our data suggest that these effects, in part, reflect the rapid control of viral replication in the lungs, which peaked between 24 and 48 hours after inoculation (hpi) and diminished within 7 days, thus creating an interval between lung inflammation and high titer antibody production in most macaques. Indeed, although SARS-CoV infection resulted in significant infiltration of macrophages in the lungs, the number of macrophages was rapidly reduced following virus clearance at $3 \mathrm{dpi}$ (Figure 5). At $7 \mathrm{dpi}$, inflammation appeared to be mostly resolved (Figure 5). By contrast, control of virus replication is less efficient in SARS patients, and the peak in viral load coincides with the first appearance of an antibody response, approximately 10 days after the onset of symptoms (6).

The low rate of ALI and interval between lung inflammation resolution and antibody production, however, makes Chinese macaques an ideal animal model for the present study. Using vaccination and passive immunization, we conditioned anti-S antibody titers in macaques during the early stage of infection when ALI was not typically observed. We found that prior administration of anti-S-IgG led to massive accumulation of monocytes/macrophages in the lungs within $2 \mathrm{dpi}$. This result is consistent with previous reports, in which mice given the SARS vaccine exhibited an immunopathologic lung reaction after the subsequent challenge with SARS-CoV $(18,19)$. Moreover, in SARS patients, clinical course and outcome are more favorable in children younger than 12 years of age compared with adolescents and adults (44), indicating that prior alternative $\mathrm{CoV}$ infection might 
A
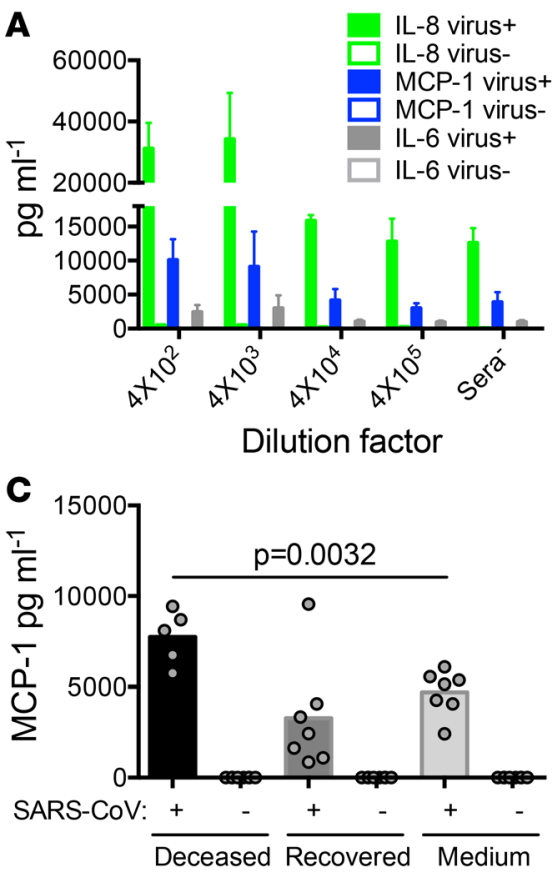

$\mathbf{E}$

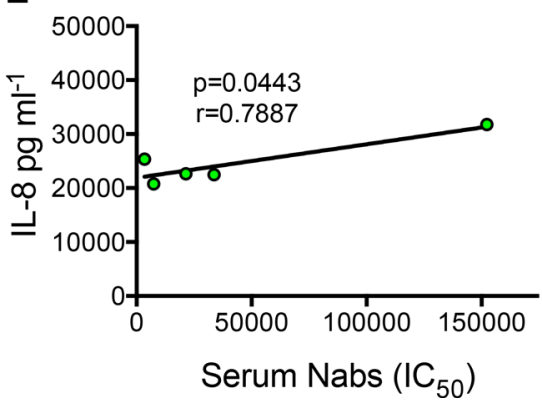

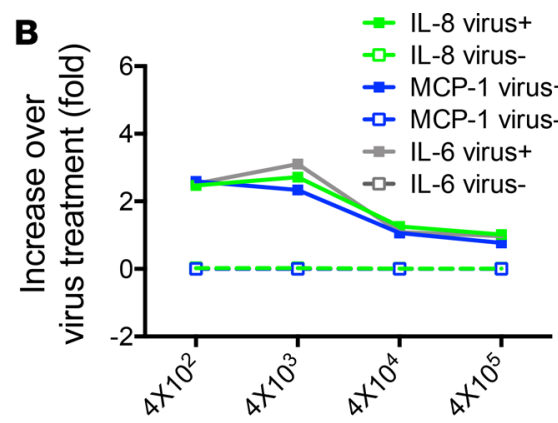

Dilution factor

D

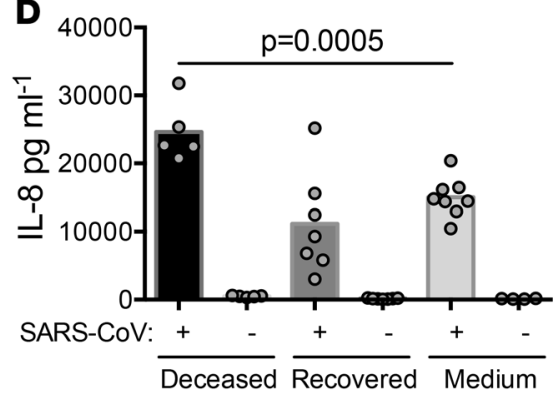

$\mathbf{F}$

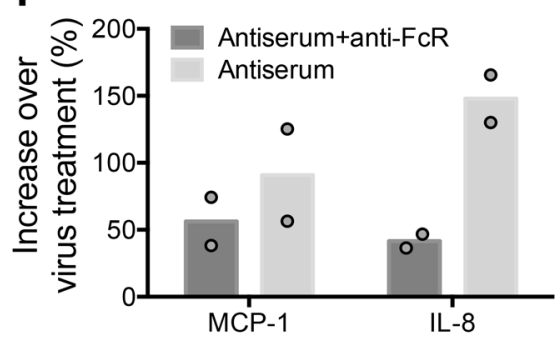

Figure 8. Sera from deceased SARS patients skewed wound-healing macrophage response partially through Fc $\gamma$ R. In vitro-polarized alternatively activated MDM were either left unstimulated or were incubated with SARS-CoV pseudovirus alone or cocultured with SARS-CoV pseudovirus and 1-400,000 serials diluted or 1/4,000 diluted sera from deceased $(n=$ 5 ) or recovered SARS patients $(n=7)$ for 20 hours. Cells treated with sera from patients alone served as controls. Secreted cytokine and chemokine levels were measured in the culture supernatant and are shown in data represented as a column graph (A, C, and $\mathbf{D})$ and fold or percentage over supernatants from macrophages treated with virus alone (B and $\mathbf{F})$. A and $\mathbf{B}$ show that addition of sera from deceased patient (D1) dose-dependently enhanced production of IL-8, IL-6, and MCP1 by SARS-CoV-treated alternatively activated MDM. $\mathbf{C}$ and $\mathbf{D}$ show significantly enhanced IL- 8 and MCP1 production by alternatively activated macrophages treated with sera from deceased SARS patients and virus compared with cells treated with virus alone. Sera treatment alone did not induce IL-8 or MCP1 production. (E) Correlation of IL-8 production with NAb titers of sera of deceased patients. Solid lines denote the relationship between histopathology scores and serum neutralizing activity. (F) Blockade of human Fc $\gamma$ R significantly reduced antiserum-enhanced IL-8 secretion and partially reduced MCP1 secretion. In vitro-polarized alternatively activated human monocyte-derived macrophages were cocultured with SARS-CoV pseudovirus and 1-4,000 diluted sera from deceased patient (D1) with or without Fc $\gamma R$ blocking antibody for 20 hours. Secreted cytokine and chemokine levels were measured in the culture supernatant and are shown in data represented as increase over supernatants from macrophages treated with virus alone. Data represent mean values or mean values \pm SEM of at least 3 independent experiments. Two-tailed unpaired Student's $t$ test and Spearman's rank correlation test were used for statistical analysis.

play a role in driving enhanced pulmonary inflammation. A recent study reported that pathogenic immune complexes promoted lung injury during the $2009 \mathrm{H} 1 \mathrm{~N} 1$ pandemic (16). It remains unknown whether immune complexes may also play a role in driving ALI during SARS-CoV infection.

Detailed analyses of macrophage heterogeneity at different stages of infection and in lungs with severe injury or mild lesion revealed distinct roles for proinflammatory and wound-healing macrophages in SARS disease progression. We observed that the early transition of macrophage responses from proinflammatory 
to wound healing with increased TGF- $\beta$ expression is associated with inflammation resolution and mild lung injury. By contrast, abrogated wound healing by S-IgG resulted in decreased TGF- $\beta$ production and prolonged classical macrophage activation, and it promoted severe lung injury. Our findings are primarily based on imaging of formalin-fixed tissues of infected macaques, which may have technical limitations. A detailed flow cytometric analysis would be helpful to further characterize macrophage subpopulations in infected lungs. Unfortunately, we were not allowed to obtain any live cells, including macrophages, for analysis by flow cytometry outside the BSL-3 laboratory in China, which is in line with "WHO biosafety guidelines for handling of SARS specimens" (https://www.who.int/csr/sars/biosafety2003_04_25/en/). Having said this, TGF- $\beta$ is mainly derived from wound-healing macrophages but not from classically activated macrophages. Therefore, by staining both TGF- $\beta$ and CD206 (another biomarker of wound-healing macrophages), we believe that the finding of reduction of this type of macrophages in the S-IgG-treated lungs is convincing.

Patients who received convalescent plasma from recovered SARS patients had shorter hospital stays and lower mortality rates without evident adverse effects (45-47). These results might reflect a different quality of antibodies in recovered patients from deceased patients during early infection. Indeed, we previously observed that deceased patients had significantly higher NAbs with a very low level of anti-N antibodies in serum during the early stage of infection (14). In contrast, recovered patients had more rapid anti-N antibody and slower NAb development (14). Consistently, serum from deceased patients - but not SARS survivors during early stages of infection - enhanced IL-6, IL-8, and MCP1 production by wound-healing macrophages (Figure 8). We previously identified epitopes in S protein that elicited NAbs and antibodies that enhanced SARS$\mathrm{CoV}$ infection $(48,49)$. Further studies that will attempt to identify antibodies and epitopes that mediate prevention or enhancement of ALI might be needed for future vaccine design and immunotherapy.

Lastly, blockade of FcyRs reduced proinflammatory cytokine production, suggesting a potential role of $\mathrm{F} c \gamma \mathrm{Rs}$ for the postulated reprogramming of alternatively activated macrophage. Fc $\gamma$ Rs are functionally divided into activating and inhibitory receptors. Activating Fc $\gamma$ Rs, such as Fc $\gamma$ RI, Fc $\gamma$ RIIA, and Fc $\gamma$ RIII, triggers production of proinflammatory chemokines and cytokines through an immunoreceptor tyrosine-based activation motif (ITAM) in their intracytoplasmic domain and SRC family kinases and spleen tyrosine kinase (SYK), whereas the inhibitory Fc $\gamma$ R, Fc $\gamma$ RIIB, has an immunoreceptor tyrosine-based inhibition motif (ITIM) in its intracytoplasmic domain and counteracts the signals that are mediated by activating Fc $\gamma$ Rs. Based on literature and our experiments, human monocyte-derived wound-healing macrophages express higher levels of CD16 (Fc $\gamma$ RIII) and CD32A (Fc $\gamma$ RIIA) (50). Therefore, it is likely that S-IgG promoted proinflammatory cytokine production through Fc $\gamma$ RI and/or Fc $\gamma$ RIIA. S-IgG did not affect classically activated macrophage function in vitro. This difference can be possibly explained by the high baseline level of proinflammatory cytokines before infection and the downregulated expression of Fc $\gamma$ R on classically activated MDM by SARS-CoV treatment.

\section{Methods}

Immunization of animals. We vaccinated Chinese rhesus macaques as previously described (51). Briefly, 16 Chinese rhesus monkeys were immunized twice on days 0 and 28. Eight of these animals received ADSMVA, whereas the other 8 animals received ADC-MVA. ADS-MVA and ADC-MVA vectors encoding SARS-CoV S protein or HCV E1E2 were constructed and produced as previously described (51). The dose for both immunizations was $5 \times 10^{8} \mathrm{TCID}_{50}$. The vaccine strain SARS-CoV HKU39849 (AY278491) shares $100 \%$ sequence homology of the $\mathrm{S}$ gene with the challenge strain SARS- $\mathrm{CoV}_{\text {PUMC }}$ (AY350750).

Animals, tissues, and SARS-CoV infection. SARS-CoV-infected animals and control animals were all adult female Chinese rhesus macaques. A total of 42 Chinese rhesus macaques were challenged i.n. with a live pathogenic SARS-CoV (PUM, TCID $\left._{50}=50\right)$ as previously described (25). An additional 3 uninfected macaques were inoculated with PBS as negative controls. Animals were subjected to daily measurement of anal temperature, routine blood assays, and chest radiography. Animals were euthanized at 2, 3, 7, 21, and 35 days after inoculation (Figure 1, Figure 2, and Figure 6). All animals were humanely euthanized with an i.v. overdose of pentobarbital and were immediately necropsied. Lung tissues (left and right cranial, middle and caudal lobes, and right accessory lobe) were collected and fixed in 10\% neutral-buffered formalin, embedded in paraffin, and sectioned at $5 \mu \mathrm{m}$

Passive transfer of IgG. SARS-CoV S protein-immune serum was generated by vaccination with ADSMVA vectors. IgG was purified from pooled sera by protein $G$ affinity chromatography to $>95 \%$ purity. IgG was depyrogenated, concentrated to $20-30 \mathrm{mg}$ protein $\mathrm{ml}^{-1}$, and administered i.v. Recipients received 200 mg or $5 \mathrm{mg}$ of purified IgG per kg body weight at 48 hours before the viral challenge. 
Neutralization. A neutralization assay was established to determine the humoral immune responses generated by the vaccine as previously described (51). The neutralizing activity of heat-inactivated animal sera was determined using a pseudotype viral entry assay. The pseudotype virus was generated through cotransfection of 293T cells with 2 plasmids, pcDNA-Sopt9 and pNL4-3Luc_Env_Vpr_, carrying the optimized S gene and a human immunodeficiency virus type 1 backbone, respectively. The serial diluted serum samples were incubated with equal amounts of pseudotype virus at $37^{\circ} \mathrm{C}$ for 1 hour. The serum-virus mixtures were subsequently added into preseeded HEK 293T-ACE2 cells. After 56 hours, infected cells were lysed to measure luciferase activity.

Nested RT-PCR and isolation of SARS-CoV. Pharyngeal swab samples were collected from infected monkeys on the first day after inoculation and subsequently tested for SARS-CoV using nested RT-PCR as previously described (25). Briefly, RNA was isolated using TRIZOL (Invitrogen). RT-PCR was performed in a $50 \mu 1$ reaction volume with outer primer pair (5'-GCTGCATTGGTTTGTTATATCGTTA-TGC-3') and (5'-ATACAGAATACAT-AGATTGCTGTTATCC-3'), inner primer pair (5'-TCACTTGCTTCCGTTGAGGTAGC-CAGCGTGGT-GGTTCATACAA-3') and (5'-GGTTTCGGATGTTACAGCGTCTCCCGG-CAGAAAGCTGTAAGCT-3'). All PCR products were verified by nucleotide sequencing. SARS-CoV was isolated as previously described. Briefly, pharyngeal swab samples from the infected macaques were inoculated onto Vero-E6 cells and cultured in DMEM (Thermo Fisher Scientific). Both cytopathic effect (CPE) and immunofluorescence assay (IFA) (incubation with 1:10 dilution of SARS patient serum) were used to determine the infection status of the animals.

Double and triple immunofluorescence staining on paraffin sections. The samples were deparaffinized and rehydrated. After blocking with normal goat serum for 30 minutes at room temperature, a rabbit antiSARS-CoV nucleocapsid antibody (ss-006-0100, eENZYME) was applied at $4^{\circ} \mathrm{C}$ overnight, followed by an Alexa Fluor 448 conjugated goat anti-rabbit IgG antibody for 1 hour at room temperature. Additional immunofluorescence staining was subsequently performed by overnight incubation with primary antibodies, followed by the detection of signals using appropriate fluorescent secondary antibodies (MilliporeSigma) for 1 hour at room temperature.

Primary antibodies for immunofluresence staining. The following primary antibodies were used in this study: SARS-CoV NP (ss-006-0100, eENZYME); MAC387 (clone MAC387, AbD, Serotec); CD68 (clone KPI, DAKO; ab125047, Abcam); CD163 (clone EDHu1, AbD and ab183476, Abcam); HAM56 (clone HAM56, DAKO); CD206 (HPA004114, MilliporeSigma); TGF- $\beta$ (sc-146, Santa Cruz Biotechnology Inc.); IL-6 (sc-1265, Santa Cruz Biotechnology Inc.); AE1/AE3 (clone AE1/AE3, DAKO).

ISH studies. ISH was developed for the detection of SARS-CoV. DNA fragments of $\mathrm{N}$ and S proteins from the SARS-CoV strain from Hong Kong (AY278491) were used as templates to generate RNA probes. The probes were labeled with digoxigenin (GENEWIZ Inc.). After deparaffinization and rehydration, the sections were treated with microwave heating in an $800 \mathrm{~W}$ domestic microwave oven (NN-ST556M, Panasonic) at medium power for 10 minutes in $0.01 \mathrm{M}$ sodium citrate buffer. Hybridization was performed at high stringency with $150 \mathrm{ng} / \mathrm{ml}$ of denatured probe at $45^{\circ} \mathrm{C}$ for 16 hours in $50 \mu 1$ of hybridization mix ( $50 \%$ formamide, $10 \%$ dextran sulfate in $2 \times$ saline sodium citrate [SSC]) in a moist chamber. Excess probe was removed by washing twice in $2 \times$ SSC for 20 minutes at $45^{\circ} \mathrm{C}$, followed by 2 washes in $1 \times$ SSC and $0.1 \times \mathrm{SSC}$ for 20 minutes at $45^{\circ} \mathrm{C}$. Immunological detection was conducted using the DIG Nuclear Acid Detection Kit (Roche Diagnostics) according to the manufacturer's instructions. Briefly, after incubation with $1 \%$ blocking reagent for 30 minutes, the sections were incubated with sheep anti-digoxigenin alkaline phosphatase conjugate (11093274910, Roche Diagnostics) diluted at 1: 500 for 2 hours at room temperature. After 3 separate washes with washing buffer (0.1 M maleic acid, $0.15 \mathrm{M} \mathrm{NaCl}$ [pH 7.5], 0.3\% Tween 20), the signal was developed using $200 \mu 1$ of a HNPP/Fast Red TR mix (1758888, Roche Diagnostics) in the dark for 45 minutes. The sections were counterstained with DAPI. Tissue sections incubated with sense RNA probe were used as a negative control.

Confocal microscopy. Confocal microscopy was performed using a Carl Zeiss LSM 700 confocal microscope equipped with 4 lasers (Zeiss). ZEN (Zeiss) was used to assign colors to the 4 channels collected: Alexa Fluor 568 and HNPP/Fast Red, which fluoresces when exposed to a 568-nm wavelength laser and appears red; Alexa Fluor 488 (Molecular Probes), which appears green; Alexa Fluor 647 (Molecular Probes), which appears cyan; and the differential interference contrast (DIC) image, which is in gray scale. The 4 channels were simultaneously collected. To differentiate between individual cells, Hoechst 33258 (nuclear marker; Molecular Probes) was used at $1 \mu \mathrm{g} / \mathrm{ml}$ and incubated for 5 minutes, followed by rinsing with water. The colocalization of antigens was demonstrated by the addition of colors as indicated in the figure legend. 
Monocyte isolation, differentiation, and polarization of macrophages. Monocytes were purified from peripheral blood mononuclear cells obtained from blood buffy coats (provided by Red Cross, Hong Kong, China) or monkey peripheral blood, as we previously described (52). Monkey peripheral blood was obtained in heparinized vacutainer collection tubes from healthy female rhesus monkeys. To generate M1 macrophages, monocytes were differentiated in RPMI 1640 medium (Thermo Fisher Scientific) supplemented with GM-CSF (400 IU/ml), 2 mM glutamine, 10\% decomplemented FCS, $100 \mathrm{IU} /$ $\mathrm{ml}$ penicillin, and $100 \mu \mathrm{g} / \mathrm{ml}$ streptomycin (Thermo Fisher Scientific) for 4 days, followed by exposure to fresh medium supplemented with 5\% FCS and containing GM-CSF and LPS (100 ng/ml) + IFN- $\gamma$ (20 ng/ml) for an additional 72 hours. To generate M2 macrophages, monocytes were differentiated in RPMI 1640 medium (Thermo Fisher Scientific) supplemented with M-CSF (50 ng/ml), $2 \mathrm{mM}$ glutamine, 10\% decomplemented FCS, $100 \mathrm{IU} / \mathrm{ml}$ penicillin, and $100 \mu \mathrm{g} / \mathrm{ml}$ streptomycin for 4 days, followed by exposure to fresh medium supplemented with $5 \%$ FCS and containing M-CSF (50 ng/ml) + IL-4 (20 ng/ml) for an additional 72 hours.

Quantification of cytokine levels. A panel of 13 cytokines, including IL-1 $\beta$, IFN- $\alpha$, IFN- $\gamma$, TNF- $\alpha$, MCP1, IL-6, IL-8, IL-10, IL-12p70, IL-17A, IL-18, IL-23, and IL-33 in monkey sera and in culture medium were quantified using multiplexing laser bead technology (BioLegend). To assess the cytokine production of macrophages, in vitro-polarized cells were washed 3 times with PBS and were subsequently activated with SARS-CoV pseudotype virus in RPMI 1640 medium (Thermo Fisher Scientific) with or without administration of highly diluted and heat-inactivated $\left(56^{\circ} \mathrm{C}\right)$ sera from macaques or SARS patients. Fc $\gamma$ R-specific mouse monoclonal antibody (5 $\mu$ g, clone 3G8, anti-hCD16, 16016682; clone FLI8.26, anti-hCD32, 16032981; and clone 10.1, anti-hCD64, 16064981 [BD Pharmingen]) was used for blockade of human Fc $\gamma$ R. The supernatant of these cultures was recovered after 20 hours of stimulation, and the cytokine levels were measured and analyzed.

Statistics. All statistical analyses were performed using the 2-tailed Student's $t$ test. $P$ values less than 0.05 were considered statistically significant. Data are presented as the mean values \pm SEM of at least 3 independent experiments unless otherwise indicated.

Study approval. The experiment was conducted in a Biosafety Level 3 animal facility. Handling and experimental procedures, including injections, blood collection, virus challenge, and sacrificing were approved and performed according to the animal welfare committee on the Use of Live Animals in Teaching and Research of Institute of Laboratory Animal Science, Beijing, China. All study subjects gave informed consent, and procedures were reviewed and approved by the University of Hong Kong Research Ethics Committee.

\section{Author contributions}

ZC, LL, and QW designed the study. LL and QW analyzed the data. LL, ZC, KYY, SP, and AL contributed to drafting the manuscript. $\mathrm{ZC}$ is the principal investigator, with overall responsibility for the design of the study. QW and CQ coordinated the autopsy organization and collection of autopsy materials of Chinese macaques. QW was involved in the development and performance of the RT-PCR assay. LL, HT, TW, and KWC performed the neutralization assay. LL, XA, and AL were involved in the development of the H\&E, SARS-CoV IHC, and ISH assays. HW performed the ISH assay and the IHC staining of NP and calculation of the mean florescence intensity in the lungs. LL, HK, and KN performed the H\&E and double- or triple-IHC staining. JF and HT performed culture of monocyte-derived macrophages. QL, HT, JP, and ZT performed analysis of cytokine production in cell culture supernatant and monkey sera. KHC and KYY coordinated the collection of sera and tissue sample from SARS patients. All authors were involved in the correlative interpretation of the pathological and molecular data.

\section{Acknowledgments}

This work was financially supported by grants from the U.S. NIH RO1HL080211 and Hong Kong Research Grants Council TRS T11-706/18-N to ZC, the TNPRC (base grant RR00164), RO1060699 to SP, HMRF16150662, and the University Development Fund/Li Ka Shing Faculty of Medicine Matching Fund of the University of Hong Kong to its AIDS Institute and Hong Kong RGV. The authors would like to thank D.D. Ho and K. Liu for support and scientific advice, and Wenjie Yu for assistance of S-IgG and C-IgG purification. 
Address correspondence to: Zhiwei Chen or Li Liu, AIDS Institute and Department of Microbiology, State Key Laboratory of Emerging Infectious Disease, Li Ka Shing Faculty of Medicine, The University of Hong Kong, 21 Sassoon Road, Pokfulam, Hong Kong S.A.R., China. Phone: 852.28199831; Email: zchenai@hku.hk (ZC). Phone: 852.39179094; Email: liuli71@hku.hk(LL).

1. Drosten C, et al. Identification of a novel coronavirus in patients with severe acute respiratory syndrome. $N$ Engl J Med. 2003;348(20):1967-1976.

2. Ksiazek TG, et al. A novel coronavirus associated with severe acute respiratory syndrome. N Engl J Med. 2003;348(20):1953-1966.

3. Hwang DM, Chamberlain DW, Poutanen SM, Low DE, Asa SL, Butany J. Pulmonary pathology of severe acute respiratory syndrome in Toronto. Mod Pathol. 2005;18(1):1-10.

4. Peiris JS, Guan Y. Confronting SARS: a view from Hong Kong. Philos Trans R Soc Lond, B, Biol Sci. 2004;359(1447):1075-1079.

5. Nicholls J, Dong XP, Jiang G, Peiris M. SARS: clinical virology and pathogenesis. Respirology. 2003;8 Suppl:S6-S8.

6. Peiris JS, et al. Clinical progression and viral load in a community outbreak of coronavirus-associated SARS pneumonia: a prospective study. Lancet. 2003;361(9371):1767-1772.

7. Ware LB, Matthay MA. The acute respiratory distress syndrome. N Engl J Med. 2000;342(18):1334-1349.

8. Herold S, Mayer K, Lohmeyer J. Acute lung injury: how macrophages orchestrate resolution of inflammation and tissue repair. Front Immunol. 2011;2:65.

9. Murray PJ, Wynn TA. Protective and pathogenic functions of macrophage subsets. Nat Rev Immunol. 2011;11(11):723-737.

10. Mosser DM, Edwards JP. Exploring the full spectrum of macrophage activation. Nat Rev Immunol. 2008;8(12):958-969.

11. Wynn TA, Vannella KM. Macrophages in Tissue Repair, Regeneration, and Fibrosis. Immunity. 2016;44(3):450-462.

12. Perlman S, Dandekar AA. Immunopathogenesis of coronavirus infections: implications for SARS. Nat Rev Immunol. 2005;5(12):917-927.

13. Channappanavar R, et al. Dysregulated Type I Interferon and Inflammatory Monocyte-Macrophage Responses Cause Lethal Pneumonia in SARS-CoV-Infected Mice. Cell Host Microbe. 2016;19(2):181-193.

14. Zhang L, et al. Antibody responses against SARS coronavirus are correlated with disease outcome of infected individuals. J Med Virol. 2006;78(1):1-8.

15. To KK, et al. High titer and avidity of nonneutralizing antibodies against influenza vaccine antigen are associated with severe influenza. Clin Vaccine Immunol. 2012;19(7):1012-1018.

16. Monsalvo AC, et al. Severe pandemic 2009 H1N1 influenza disease due to pathogenic immune complexes. Nat Med. 2011;17(2):195-199.

17. Clay C, et al. Primary severe acute respiratory syndrome coronavirus infection limits replication but not lung inflammation upon homologous rechallenge. J Virol. 2012;86(8):4234-4244.

18. Bolles $\mathrm{M}$, et al. A double-inactivated severe acute respiratory syndrome coronavirus vaccine provides incomplete protection in mice and induces increased eosinophilic proinflammatory pulmonary response upon challenge. $J$ Virol. 2011;85(23):12201-12215.

19. Tseng CT, et al. Immunization with SARS coronavirus vaccines leads to pulmonary immunopathology on challenge with the SARS virus. PLoS ONE. 2012;7(4):e35421.

20. Zhao J, et al. Airway Memory CD4(+) T Cells Mediate Protective Immunity against Emerging Respiratory Coronaviruses. Immunity. 2016;44(6):1379-1391.

21. Channappanavar R, Fett C, Zhao J, Meyerholz DK, Perlman S. Virus-specific memory CD8 T cells provide substantial protection from lethal severe acute respiratory syndrome coronavirus infection. J Virol. 2014;88(19):11034-11044.

22. Zhao J, Zhao J, Perlman S. T cell responses are required for protection from clinical disease and for virus clearance in severe acute respiratory syndrome coronavirus-infected mice. J Virol. 2010;84(18):9318-9325.

23. Honda-Okubo Y, Barnard D, Ong CH, Peng BH, Tseng CT, Petrovsky N. Severe acute respiratory syndrome-associated coronavirus vaccines formulated with delta inulin adjuvants provide enhanced protection while ameliorating lung eosinophilic immunopathology. J Virol. 2015;89(6):2995-3007.

24. Chen Z, et al. Recombinant modified vaccinia virus Ankara expressing the spike glycoprotein of severe acute respiratory syndrome coronavirus induces protective neutralizing antibodies primarily targeting the receptor binding region. $J$ Virol. 2005;79(5):2678-2688.

25. Liu L, et al. Spatiotemporal interplay of severe acute respiratory syndrome coronavirus and respiratory mucosal cells drives viral dissemination in rhesus macaques. Mucosal Immunol. 2016;9(4):1089-1101.

26. Li BJ, et al. Using siRNA in prophylactic and therapeutic regimens against SARS coronavirus in Rhesus macaque. Nat Med. 2005;11(9):944-951.

27. Qin C, et al. An animal model of SARS produced by infection of Macaca mulatta with SARS coronavirus. $J$ Pathol. 2005;206(3):251-259.

28. Soulas C, et al. Recently infiltrating MAC387(+) monocytes/macrophages a third macrophage population involved in SIV and HIV encephalitic lesion formation. Am J Pathol. 2011;178(5):2121-2135.

29. Cai Y, Sugimoto C, Arainga M, Alvarez X, Didier ES, Kuroda MJ. In vivo characterization of alveolar and interstitial lung macrophages in rhesus macaques: implications for understanding lung disease in humans. J Immunol. 2014;192(6):2821-2829.

30. Murray PJ, et al. Macrophage activation and polarization: nomenclature and experimental guidelines. Immunity. 2014;41(1):14-20.

31. Imai Y, et al. Identification of oxidative stress and Toll-like receptor 4 signaling as a key pathway of acute lung injury. Cell. 2008;133(2):235-249.

32. Atreya R, et al. Blockade of interleukin 6 trans signaling suppresses T-cell resistance against apoptosis in chronic intestinal inflammation: evidence in crohn disease and experimental colitis in vivo. Nat Med. 2000;6(5):583-588.

33. Braciale TJ, Sun J, Kim TS. Regulating the adaptive immune response to respiratory virus infection. Nat Rev Immunol. 2012;12(4):295-305 
34. Subbarao K, et al. Prior infection and passive transfer of neutralizing antibody prevent replication of severe acute respiratory syndrome coronavirus in the respiratory tract of mice. J Virol. 2004;78(7):3572-3577.

35. Yang ZY, et al. A DNA vaccine induces SARS coronavirus neutralization and protective immunity in mice. Nature. 2004;428(6982):561-564.

36. Du L, et al. Priming with rAAV encoding RBD of SARS-CoV S protein and boosting with RBD-specific peptides for T cell epitopes elevated humoral and cellular immune responses against SARS-CoV infection. Vaccine. 2008;26(13):1644-1651.

37. Du L, et al. Intranasal vaccination of recombinant adeno-associated virus encoding receptor-binding domain of severe acute respiratory syndrome coronavirus (SARS-CoV) spike protein induces strong mucosal immune responses and provides long-term protection against SARS-CoV infection. J Immunol. 2008;180(2):948-956.

38. Du L, et al. Receptor-binding domain of SARS-CoV spike protein induces long-term protective immunity in an animal model. Vaccine. 2007;25(15):2832-2838.

39. Fett C, DeDiego ML, Regla-Nava JA, Enjuanes L, Perlman S. Complete protection against severe acute respiratory syndrome coronavirus-mediated lethal respiratory disease in aged mice by immunization with a mouse-adapted virus lacking $\mathrm{E}$ protein J Virol. 2013;87(12):6551-6559.

40. Iwata-Yoshikawa N, et al. Effects of Toll-like receptor stimulation on eosinophilic infiltration in lungs of BALB/c mice immunized with UV-inactivated severe acute respiratory syndrome-related coronavirus vaccine. J Virol. 2014;88(15):8597-8614.

41. Qin E, et al. Immunogenicity and protective efficacy in monkeys of purified inactivated Vero-cell SARS vaccine. Vaccine. 2006;24(7):1028-1034

42. Zhou J, et al. Immunogenicity, safety, and protective efficacy of an inactivated SARS-associated coronavirus vaccine in rhesus monkeys. Vaccine. 2005;23(24):3202-3209.

43. Openshaw PJ, Tregoning JS. Immune responses and disease enhancement during respiratory syncytial virus infection. Clin Microbiol Rev. 2005;18(3):541-555.

44. Stockman LJ, et al. Severe acute respiratory syndrome in children. Pediatr Infect Dis J. 2007;26(1):68-74

45. Mair-Jenkins J, et al. The effectiveness of convalescent plasma and hyperimmune immunoglobulin for the treatment of severe acute respiratory infections of viral etiology: a systematic review and exploratory meta-analysis. J Infect Dis. 2015;211(1):80-90.

46. Cheng Y, et al. Use of convalescent plasma therapy in SARS patients in Hong Kong. Eur J Clin Microbiol Infect Dis. 2005;24(1):44-46.

47. Soo YO, et al. Retrospective comparison of convalescent plasma with continuing high-dose methylprednisolone treatment in SARS patients. Clin Microbiol Infect. 2004;10(7):676-678.

48. Wang Q, et al. Immunodominant SARS Coronavirus Epitopes in Humans Elicited both Enhancing and Neutralizing Effects on Infection in Non-human Primates. ACS Infect Dis. 2016;2(5):361-376.

49. Yi CE, Ba L, Zhang L, Ho DD, Chen Z. Single amino acid substitutions in the severe acute respiratory syndrome coronavirus spike glycoprotein determine viral entry and immunogenicity of a major neutralizing domain. J Virol. 2005;79(18):11638-11646.

50. Pahl JH, et al. Macrophages inhibit human osteosarcoma cell growth after activation with the bacterial cell wall derivative liposomal muramyl tripeptide in combination with interferon- $\gamma$. J Exp Clin Cancer Res. 2014;33:27.

51. Kieran J, et al. The relative efficacy of boceprevir and telaprevir in the treatment of hepatitis C virus genotype 1 . Clin Infect Dis 2013;56(2):228-235

52. Cheng L, et al. Monoclonal antibodies specific to human $\triangle 42 \mathrm{PD} 1$ : A novel immunoregulator potentially involved in HIV-1 and tumor pathogenesis. MAbs. 2015;7(3):620-629. 